\title{
Defining Monocyte Differentiation into Colonic and Ileal Macrophages
}

Mor Gross-Vered ${ }^{1}$, Sébastien Trzebanski ${ }^{1}$, Anat Shemer ${ }^{1}$, Biana Bernshtein ${ }^{1}$, Caterina Curato $^{1}$, Gil Stelzer ${ }^{2}$, Tomer-Meir Salame ${ }^{2}$, Eyal David ${ }^{1}$, Sigalit Boura-Halfon ${ }^{1}$, Louise Chappell-Maor ${ }^{1}$, Dena Levkovits ${ }^{2}$ and Steffen Jung ${ }^{1^{*} \dagger}$

${ }^{1}$ Departments of Immunology and ${ }^{2}$ Life Science Core Facilities, Weizmann Institute of Science, Rehovot 76100, Israel.

- $\quad$ Lead contact : s.jung@weizmann.ac.il (S.J.)

${ }^{\dagger}$ Corresponding author e-mail: s.jung@weizmann.ac.il (S.J.) 


\begin{abstract}
Monocytes are circulating short-lived macrophage precursors that are recruited on demand from the blood to sites of inflammation and challenge. In steady state, classical monocytes give rise to vasculature-resident cells that patrol the luminal side of the endothelium. In addition, classical monocytes feed macrophage compartments of selected organs, including barrier tissues, such as the skin and intestine, as well as the heart. Monocyte differentiation under conditions of inflammation has been studied in considerable detail. In contrast, monocyte differentiation under non-inflammatory conditions remains less well understood. Here we took advantage of a combination of cell ablation and precursor engraftment to investigate the generation of gut macrophages from monocytes. Collectively, we identify factors associated with the gradual adaptation of monocytes to tissue residency. Moreover, comparison of monocyte differentiation into the colon and ileum-resident macrophages revealed the graduated acquisition of gut segment-specific gene expression signatures.
\end{abstract}




\section{Introduction}

The recent past has seen major advance in our understanding of the diverse origins of tissue macrophages, as well as their discrete maintenance strategies. Macrophages were shown to arise from three distinct developmental pathways that differentially contribute to the tissue compartments in the embryo and adult ${ }^{1}$. In the mouse, embryonic tissue macrophages first develop from primitive macrophage progenitors that originate in the yolk sac (YS). In the brain, YS-macrophage-derived macrophages persist throughout adulthood, while in most other tissues these cells are replaced by fetal monocytes that derive from multi-potent erythro-myeloid progenitors (EMP). Definitive hematopoiesis commences from E10.5 with the generation of hematopoietic stem cells (HSC) that first also locate to the fetal liver, but eventually seed the bone marrow (BM) to maintain adult hematopoiesis. Most EMP-derived tissue macrophage compartments persevere throughout adulthood without major input from HSC-derived cells; however, in certain barrier tissues, as well as selected other organs, like the heart, embryonic macrophages are progressively replaced by HSC-derived cells involving a blood monocyte intermediate ${ }^{2}$.

Monocytes are continuously generated in the BM involving a sequence of developmental intermediates, before extravasation into the circulation ${ }^{3,4}$. Once in the blood, murine classical Ly6 $\mathrm{C}^{+}$monocytes have a limited half-life ${ }^{5}$. On demand, $\mathrm{Ly}_{6 \mathrm{C}} \mathrm{C}^{+}$monocytes can be rapidly recruited to sites of injury and challenge, where they complement tissueresident macrophages and dendritic cells. In absence of challenge, some $\mathrm{Ly}_{6 \mathrm{C}^{+}}$monocytes give rise to vasculature-resident $\mathrm{Ly} \mathrm{C}^{10}$ cells, which patrol the vessel walls ${ }^{6}$. These cells display macrophage characteristics including extended life spans ${ }^{5,7}$. In addition, Ly6C ${ }^{+}$ monocytes replenish the above-mentioned selected steady state tissue macrophage compartments, including the gut and skin ${ }^{3}$. Given their mobility, plasticity and key role in pathologies, the manipulation of monocytes and their differentiation could bear considerable therapeutic potential. However, monocyte differentiation into tissue resident cells remains incompletely understood.

Gut macrophages, which reside in the connective tissue underlying the gut epithelium, the lamina propria, are considered key players for the maintenance of intestinal homeostasis. As such, they constantly sense their environment and respond to the unique microbiota and food challenge ${ }^{8,9}$. Recent studies revealed that monocyte-derived lamina propria macrophages comprise in mice two populations, i.e. short-lived cells and long-lived cells with self-renewing capacity ${ }^{10}$; the latter population might also include remnants of embryonic populations, as could additional intestinal long-lived macrophage populations that reside near blood vessels, nerves and in the Peyer's patches ${ }^{11}$. Evidence for macrophages with different half-lives is also emerging for the human small intestine ${ }^{12}$. Collectively, these 
findings highlight the existence of considerable macrophage heterogeneity, not only between different organs, but also within given tissues.

Monocyte differentiation into intestinal macrophages involves phenotypic changes with respect to $\mathrm{Ly} 6 \mathrm{C}, \mathrm{CD} 64$ and $\mathrm{MHCll}$ expression, a sequence described as 'monocyte waterfall' ${ }^{13}$. Mature steady-state gut macrophages tolerate the commensal microbiota and food antigens ${ }^{8,9}$. Their relative unresponsiveness is thought to rely on regulatory circuits that balance the expression of pro- and anti-inflammatory gene products, such as cytokines and molecules participating in pattern recognition receptor signaling cascades.

Macrophages located in different tissues display characteristic enhancer landscapes and gene expression profiles, which have been attributed to the exposure of instructing factors of the microenvironment they reside in ${ }^{14}$. Gut segments display distinct anatomy, function and microbiota load ${ }^{15}$ and macrophages of small and large intestine hence also likely differ. Despite the known distinct susceptibility of the colon and ileum to pathology, such as to the IL10R deficiency ${ }^{16,17}$ and in ulcerative colitis, so far no comparative analysis of their macrophages has been reported. Likewise, our general understanding of monocytederived tissue resident macrophages remains scarce and is largely restricted to settings of inflammation.

Here we investigated monocyte differentiation into intestinal macrophages in the small and large intestine. Using adoptive monocyte transfers into macrophage-depleted recipients ${ }^{18,19}$, we synchronized the macrophages in terms of development, isolated colonic and ileal macrophages and subjected them to transcriptome profiling. Our data establish the distinct identities of gut segment resident macrophages and shed light on the kinetics and gradual gene expression of specific factors for their establishment of their identities.

\section{Results}

\section{Monocyte transcriptomes acquired during differentiation into ileal and colonic gut macrophages}

Tissue macrophages display distinct gene expression profiles and enhancer landscapes ${ }^{14}$. This holds also for intestinal macrophages residing in small and large intestine. Transcriptomes of Ly6C ${ }^{+} \mathrm{BM}$ monocytes, i.e. the macrophage precursors, and transcriptomes of their progeny in colon and ileum displayed 6200 genes differentially expressed at least 2-fold across all analyzed data sets out of a total of 12345 detected genes (Suppl. Fig. 1A-C). 2255 genes were expressed in monocytes and down-regulated in macrophages (cluster I). Conversely, cluster II comprised genes whose expression was absent from monocytes, but shared by both small and large intestinal macrophages. Finally, 
1087 and 987 genes were either preferentially or exclusively expressed in ileal or colonic macrophages, respectively.

To further characterize adult monocyte-derived gut macrophages, we took advantage of an experimental system involving monocyte engraftment of macrophage-depleted animals 18,19. Analysis of transferred cells at different intervals from engraftment allows the study of intestinal macrophage development over time, since monocyte differentiation is synchronized. For the cell ablation we used [CD11c-DTR > WT] BM chimeras, in which diphtheria toxin (DTx) receptor (DTR) transgenic intestinal macrophages can be conditionally ablated by DTx injection ${ }^{18,19}$ (Fig. 1A). Two days prior to monocyte transfer, DTx was applied to the recipients to clear their intestinal macrophage niche and mice were subsequently treated with DTx every second day. The monocyte graft was isolated from BM of $\mathrm{CX}_{3} \mathrm{CR} 1^{\mathrm{GFP} /+}$ mice ${ }^{20}$ and defined as CD117- CD $11 \mathrm{~b}^{+} \mathrm{CD} 115^{+} \mathrm{Ly}^{-} \mathrm{C}^{+} \mathrm{GFP}^{\text {int }}$ cells; donor animals also harbored an allotypic marker (CD45.1) (Fig. 1A, Suppl. Fig. 1A). Grafted cells could be visualized in recipient gut tissue and underwent expansion, as reported earlier ${ }^{19}$ (Suppl. Fig. 2). Intestinal tissues of recipient mice were harvested on day 4, 8 and 12 post engraftment (Fig. 1A) to isolate graft-derived macrophages according to CD45.1 and $\mathrm{CX}_{3} \mathrm{CR} 1 /$ GFP expression; these cells were CD11 $\mathrm{b}^{+} \mathrm{CD} 4^{+} \mathrm{Ly} \mathrm{C}^{-}$and mainly CD11 $\mathrm{c}^{\text {hi }}$ (Fig. 1B). Of note, specifics of the system preclude harvest of graft-derived cells at later time points ${ }^{19}$. The monocyte graft and colonic and ileal macrophage populations were subjected to bulk RNA-seq using MARS-Seq technology ${ }^{21}$.

Gene expression profiling revealed robust changes already at day 4 post engraftment; changes appeared to be tissue- rather than time-specific, with the exception of two day 4 samples of ileal macrophages, which clustered with the colonic samples (Fig. 1C, Suppl. Fig. 3A). Transcriptomes of the retrieved engrafted macrophages lacked expression of pro-inflammatory genes, such as Saa3, Lcn2, II1b, II6 and Tnf, as opposed to cells retrieved from colitic animals treated with Dextran Sulfate Sodium (DSS) ${ }^{22}$ (Suppl. Fig. 4). This supports the earlier notion that the cell ablation results in a transient tissue response, but the monocyte transfer system mimics cell differentiation close to steady-state conditions 19

Comparative transcriptome analysis of grafted monocytes, their progeny retrieved from the recipient intestines, and resident ileal and colonic macrophages that were independently retrieved from $\mathrm{CX}_{3} \mathrm{CR} 1^{+/ G F P}$ mice revealed 4213 differentially expressed genes (DEG) (>4-fold differences in any pairwise comparison among a total of 12878 genes) (Fig. 1D). 748 genes were exclusively expressed in the monocyte graft, including $L y 6 c 2, C c / 6$ and Cebpe (Cluster I) (Fig. 1D, E). 793 genes shared expression in graft-derived cells and gut macrophages in colon and ileum (Cluster II). These included Irf6, Gata6, Ly6A and Smad7. Cluster III comprised 634 genes that were either exclusively or preferentially expressed in 
colonic macrophages, such as Pparg, Foxa1 and TIr1 (Fig. 1E). Cluster IV comprised 539 genes exclusively or preferentially expressed in ileal macrophages, such as Nos2, Sox4 and Mmp9 (Fig. 1E). Metascape analysis ${ }^{23}$ highlighted that genes associated with cluster II, II and IV were associated with distinct pathways, particularly with respect to epithelial cell communication and cell metabolism (Suppl. Fig. 3B-E). We also identified genes that differed in expression between engrafted and resident macrophages. Specifically, 796 and 467 genes displayed high or low expression levels in monocytes, respectively, were highly expressed in resident colonic macrophages, but either low or absent in the graft-derived cells (Cluster V and VI). Finally, cluster VII comprised 236 genes that were expressed in monocytes, and down-regulated in resident gut macrophages, but not in the grafted cells.

Volcano plot analysis revealed that early monocyte differentiation (graft vs day 4 ) was characterized by abundant changes in gene expression in both colon and ileum. In the colon 3407 genes were up- and 3608 genes were down-regulated; in the ileum 2975 genes were up- and 3206 genes were down-regulated, with up to 10 fold change (Fig. 1F). Later time points (day 4 to day 8 and day 8 to day 12) were characterized by less pronounced alterations, both with respect to the number of DEG and their fold change (Fig. 1G,H). In the colon 289 genes (66\% of all significantly-changed genes) were induced between day 4 and 8 and only 111 genes (19\%) were up-regulated between day 8 and 12. In the ileum this trend was reversed, with 95 genes (21\%) up-regulated between day 4 and 8, but 321 genes (85\%) induced between day 8 and 12. Collectively, this establishes that monocytes that enter distinct gut segments rapidly acquire characteristic transcriptomic signatures.

\section{Transcriptomes of engrafted cells differ from resident macrophages.}

Our experimental set up allows us to focus on cells that entered the gut in a defined time window. Interestingly, even by day 12 , monocyte graft-derived cells differed in expression profiles when compared to resident ileal and colonic macrophages (Fig. 2A). Differences included genes absent from, and exclusively expressed by engrafted cells (Fig. 1D clusters V - VII, Fig. 2B-D). These differences might be attributable to incomplete macrophage maturation or to the recently reported heterogeneity of the intestinal macrophage compartment ${ }^{10,11}$. Notably, the majority of the cells we retrieve likely comprise lamina propria I mucosa resident macrophages, rather than the less abundant macrophages of the submucosa or muscularis layer ${ }^{10,11}$. Generation of a CD4 ${ }^{+} \mathrm{Timd}^{+}{ }^{+}$subpopulation of lamina propria macrophages was reported to require prolonged residence in the tissue ${ }^{10}$. Although grafted cells in both colon and ileum acquired with time some expression of a hallmark of the long-lived cells, the phosphatidyl serine receptor Timd4 (Tim4) (Fig. 2B, Fig.1D cluster VI), other signature genes, such as Cd209f, C2 and Rusc2 ${ }^{10}$, were not expressed in the time frame analyzed here (Fig. 2B). The hypothesis that 12 days were insufficient for engrafted 
macrophages to acquire the full gene expression profile of resident cells was furthermore corroborated by the delayed onset of MHCII (encoded by H2-ab1) expression, one of the markers for intestinal macrophage maturation ${ }^{13,24}$ (Fig. 2C). Engrafted macrophages were also characterized by lack of expression of Irf1 and the member of the TAM receptor kinase family $A x I$ (Fig. 2C).

Genes, whose expression was low to absent in resident macrophages from both colon and ileum, but prominent in the engrafted cells (Fig.1D cluster VII), included the ones encoding ribosome-associated proteins and histones (Fig. 2D). As previously shown ${ }^{19}$, the reconstitution of emptied intestinal tissue with monocyte-derived cells involves clonal expansion, that is less likely to occur in physiological setting. In line with this notion, out of the 6383 genes significantly differing between engrafted and resident macrophages from either colon or ileum, 897 were annotated with a Gene Ontology (GO) term associated with 'proliferation' and 'cell cycle'; 99 of these DEG were low to absent in resident macrophages from both tissues retrieved from non-engrafted $\mathrm{CX}_{3} \mathrm{CR} 1^{\mathrm{GFP} /+}$ mice, such as Hmga1 (Fig. 1D).

We recently reported the requirement of IL10Ra on colonic macrophages for gut homeostasis. Mice lacking this cytokine receptor on intestinal macrophages develop severe gut inflammation in the colon, but not the ileum ${ }^{17,25}$, as do children that harbor an IL10RA deficiency ${ }^{16}$. Interestingly, II10ra was not induced in engrafted colonic macrophages, even 12 days after tissue entry, while engrafted ileal macrophages displayed //10ra transcripts as early as day 4 (Fig. 2E). Expression of the cytokine IL10 itself was almost absent from monocytes and engrafted macrophages in both tissues, but significantly present in resident macrophages retrieved from non-engrafted $\mathrm{CX}_{3} \mathrm{CR} 1^{\mathrm{GFP} /+}$ mice, to a larger extent in the colon than the ileum (Fig. 2E). Of note, $/ 11 b$ expression displayed a similar pattern in line with an earlier suggestion that IL1 might induce macrophage IL-10 expression ${ }^{26}$. Likewise, genes induced following macrophage exposure to II10, such as Ccr5 ${ }^{27}$ and Socs ${ }^{28}$ displayed similar expression patterns. This suggests that in our model the IL10/IL10R axis is inactive in newly differentiated macrophages and established only after further maturation in the tissue.

Flow cytometric analysis of colon tissue of WT C57BL/6 mice identifies a small population of $\mathrm{CD} 11 \mathrm{~b}^{+} \mathrm{Ly}_{6 \mathrm{C}^{+}} \mathrm{MHCll}^{-}$cells that likely represent recent monocyte immigrants into the tissue (Suppl. Fig 5A). These rare cells probably entered the lamina propria to maintain the steady state macrophage pool of the intestine, before differentiating and have been referred to as P1 population of a 'monocyte waterfall' ${ }^{13}$. Global RNAseq analysis of this population, alongside the $\mathrm{Ly}_{6 \mathrm{C}^{+}} \mathrm{MHCll}^{-}$macrophages revealed that, like the day $4 \mathrm{graft}$, these endogenous gut immigrants down-regulated monocyte markers and gained a gut macrophage signature, including expression of the Forkhead transcription factor (TF) FoxD2 and the nuclear receptor Nr3c2 (Suppl. Fig 5B, C). Endogenous gut immigrants did not display a signature indicating proliferation, such as Rp/10 or Hmga1. However, like the 
grafted cells, these early colonic immigrants lacked signature genes associated with longlived gut macrophages, including Timd4 and CD209f, as well as expression of I/10ra and II10.

To further corroborate our data, we used a distinct cell ablation model and performed adoptive monocyte transfers into DTx-treated [CX3CR1-DTR > WT] BM chimeras ${ }^{29,30}$. The gene lists of upregulated and down regulated genes in macrophages retrieved from engrafted [CD11C-DTR > WT] and [CX3CR1-DTR > WT] chimeras showed with $71 \%$ and $59 \%$, respectively, considerable overlap (Suppl. Fig 6). While our adoptive transfer inherently aims at the reconstitution of ablated cells, which differ in the two models, the observed coherence suggest robustness of the approach.

Collectively, these data show that despite some differences, monocyte graft-derived cells recapitulate the 'monocyte waterfall' ${ }^{13}$.

\section{Monocyte differentiation into gut segment-specific macrophages}

We next focused on factors that might be involved in the generation of segment-specific macrophages, i.e. genes whose expression differed between colonic and ileal macrophages. 458 genes were up-regulated during monocyte differentiation in a segment-specific manner 351 in colonic macrophages and 107 in ileal macrophages (Fig. 3A). Monocyte differentiation into ileal macrophages was accompanied by induction of Gata and Hbox TF family members, such as Gata5 and Hbox3 (Fig. 3A), the chemokine $\mathrm{Ccl} 5$ and the chemokine receptor CCR9 (Suppl. Table). Monocytes that entered colon tissue preferentially up-regulated Foxd2, the nuclear receptor $\mathrm{Nr} 3 \mathrm{c} 2$, and the dominant negative helix-loop-helix protein Id2 (Fig. 3A, Suppl. Table).

Of the genes, which were specifically down-regulated in only one intestinal segment, 78 genes followed this trend in colonic and 99 in ileal macrophages. The latter downregulated genes included 7 TFs, such as Foxp1 and Trim16, while Arid5a and Elk3 were specifically down-regulated in the colon (Fig. 3B).

Many genes related to immune reaction and response to challenge displayed higher expression in ileal macrophages than their colonic counterparts. Examples are: Arid5a, whose gene product regulates IL6 ${ }^{31}$; Elk3, which encodes a member of the ETS TF family and was reported to modulate the phagocytosis of bacteria by macrophages ${ }^{32}$ and Ano6, that is down-regulated in colonic macrophages (Fig. 3B), and reportedly supports microbiocidal activity of macrophages involving $\mathrm{P}_{2} \mathrm{X}_{7}$ receptor signaling ${ }^{33}$. In contrast, the enzyme Sod1, which was reported to impair macrophage-related parasite killing in cutaneous Leishmaniasis ${ }^{34}$, showed lower expression in ileal macrophages (Fig. 3B) Another interesting group of genes are those, which are expressed in monocytes, but further up-regulated in one tissue upon differentiation and down-regulated in the other. These genes 
might encode factors whose expression is incompatible with segment-specific macrophage fates. 54 such genes were expressed in colonic engrafted macrophages and silenced in their ileal counterparts; 15 genes followed an opposite trend (Fig. 3C). Only one TF was found in both groups, KLF4 (Fig. 3C). Aldh2 encoded by Aldh2, mostly known for its role in alcohol detoxification, was recently reported to play a role in repression of ATP6V0E2, which is critical for proper lysosomal function, autophagy, and degradation of oxidized LDL ${ }^{35}$ (Fig. 3C). Hdac7 encoded by $\mathrm{Hdac7}$ and up-regulated in ileal macrophages and down-regulated in colonic macrophages, was reported to interfere with the myeloid gene expression pattern and to inhibited macrophage-specific functions ${ }^{36}$ (Fig. 3C). Finally, Fmn/3 participates in filopodia generation ${ }^{37}$ (Fig. 3C).

Collectively, these data establish that monocytes establish gut segment specific gene expression patterns, likely under the influence of local cues.

\section{Monocyte differentiation into generic tissue macrophages}

Transcriptomes of colonic and ileal macrophages 4 days after monocyte tissue entry were alike, with many genes sharing expression in both tissues when compared to their monocyte progenitors. Overall, by day 4, expression of 2007 genes was down-regulated more than 2fold in the monocyte graft following differentiation into macrophages in both tissues (out of 12485 genes expressed). 2404 genes were induced in both the colon and ileum, arguably as part of a generic transcriptome signature of intestinal macrophages (Fig. 4A).

Notably, 919 of the genes induced during monocyte differentiation into generic intestinal macrophages displayed very low prior expression in monocytes - below 50 reads (Fig. 4B). In contrast, expression of fewer transcripts (183) seemed to be actively silenced during the differentiation process, as seen in the violin blot in Figure 4B. This implies that monocytes actively acquire macrophage identities by de novo mRNA synthesis, while much of the monocytic gene expression is compatible with the differentiation process.

The top $5 \mathrm{GO}$-terms associated with genes up-regulated in intestinal macrophages related to cell adhesion and migration processes (Figure 4C), including the chemokine Cxcl1, Ptprk which regulates cell contact and adhesion, and the integrin Itga6 (Fig. 4D) The top 5 GOterms associated with down-regulated genes in macrophages included cell cycle and division, as well as mRNA processing and DNA repair (Fig. 4C,D), for example DNA polymerase beta (Polb), a cell cycle checkpoint regulator (Rad17) and an RNA helicase (Setx). Collectively, these data are in line with the transformation of circulating monocytes into non-migratory tissue resident cells and suggest a role for DNA damage-associated molecules during the differentiation process.

Gene expression changes are driven by TFs. In the case of intestinal macrophages, 3 major TF families seem to participate in monocyte-macrophage differentiation: CCAAT- 
enhancer-binding proteins (C/EBPs), E2 transcription factors (E2F) and early growth response TFs (Egr). Four of the Cebp family members (Cebpa, $b, d, g$ ) and $5 \mathrm{E} 2 \mathrm{~F}$ family members $(E 2 f 2,4,6,7,8)$ were significantly down-regulated upon monocyte differentiation into macrophages (Fig. 5A). In contrast, Egr1, 2 and 3 were up-regulated. Other downregulated TFs included the regulators of immune response Bach1, Bcl6, Irf9, Nfatc3, Nfkb1 and Rela, as well as the master macrophage TF Spi1 (PU.1) (Fig. 5B). The list of induced genes was enriched with homeobox TFs such as Sox13, Pbx1, Foxa1 and others (Fig. 5C). In addition, this group comprised TFs that had previously been reported to be critical for the development of other tissue macrophages, such as the Nuclear Receptor LXR encoded by Nr1h3 for splenic macrophages and Gata6 for peritoneal macrophages ${ }^{2,38}$.

\section{Comparison of tissue-resident and vasculature-resident monocyte-derived cells}

Monocytes are generated in the BM to be subsequently disseminated throughout the body via blood vessels. Under inflammation, the cells are rapidly recruited to the site of injury. In absence of challenges, $\mathrm{Ly}_{6 \mathrm{C}^{+}}$monocytes can have distinct fates. A fraction of them gives rise to vasculature-resident $\mathrm{Ly}_{6 \mathrm{C}}$ - 'patrolling' cells ${ }^{6}$. Other cells contribute to the homeostatic replenishment of selective tissue macrophage compartments (Fig. 6A). To gauge the impact of the blood environment, as compared to a solid tissue such as the intestine, on the differentiation process, we next compared transcriptomes of Ly6C- blood cells and gut macrophages to their $L y 6 C^{+}$monocyte precursors (Suppl. Fig. 5). A heat map of all 1303 genes, whose expression significantly differed between $\mathrm{Ly}_{6 \mathrm{C}^{+}}$monocytes compared to blood- and gut tissue-resident cells (day 4), revealed 5 clusters (Fig. 6B). Monocyte progeny, whether in vasculature or tissue shared signatures, showed considerable similarities as reflected in the expression pattern of two thirds of the genes (66.7 \%). Specifically, clusters I and II comprised 780 genes that were down-regulated upon Ly6C ${ }^{+}$ monocyte differentiation, including hallmark monocyte markers, such as $L y 6 c, C c r 2$ and Mmp8 and Myd88 (Fig. 6B,C). 160 genes were induced in both blood- and tissue resident monocyte-derived cells, albeit to different extend, including as Pparg, Ets2 and Tgfbr2 (Cluster III) (Fig. 6B,D). Cluster IV and V spanned one third of the genes differentially expressed by $\mathrm{Ly} 6 \mathrm{C}^{+}$monocytes and their progenies, but distinct in tissue and vascular resident cells. Specifically, cluster IV comprised 253 genes up-regulated in blood-resident cells and down-regulated in gut macrophages. This included Csf2ra, Nfkb1, I/10ra and Spi1 (PU.1). Cluster V comprised 110 genes induced in gut macrophages but not vasculatureresident cells, such as the mitochondrial master regulator Ppargc1b and the metalloprotease Adam19 (Fig. 6B). Concerning TFs, Cebpb was induced in Ly6C- blood monocytes, as was previously reported ${ }^{7}$, while Cebpa and Cebpd were down-regulated in both blood- and gut- 
resident cells (Fig. 6E). IIf family members 7, 8 and 9 were down-regulated during Ly6C ${ }^{+}$ monocyte differentiation in blood and gut.

Collectively, these data establish that vasculature-resident Ly6C- monocytes and gut macrophages that derive both from $\mathrm{Ly}_{6 \mathrm{C}^{+}}$monocytes display considerable overlap in transcriptomic signatures, but also display gene expression patterns that are likely associated with their specific environments.

\section{Discussion}

Adult tissue macrophages can derive from distinct origins ${ }^{1,2}$. Most tissue macrophages are currently believed to be generated in the embryo from EMP via a fetal liver monocyte intermediate and subsequently maintain themselves through self-renewal. Selected macrophages residing in barrier tissues, such as gut and skin, however rely on constant replenishment from blood monocytes. Here we report the study of this macrophage generation from monocyte precursors.

Following tissue damage and infection, classical monocytes, defined as CD $14^{+}$cells in humans, and $\mathrm{Ly}_{6 \mathrm{C}} \mathrm{C}^{+}$cells in mice critically contribute to inflammatory reactions by promoting and resolving acute challenges ${ }^{3,4}$. At the sites of injury, monocytes can give rise to cells with both macrophage and DC features. Monocyte differentiation during inflammation has been studied in various pathophysiological settings, including experimental auto-immune encephalitis ${ }^{39} 31$, colitis ${ }^{40,41}$ and others ${ }^{42}$. To study less well understood physiological monocyte differentiation in absence of overt inflammation, we took advantage of an experimental system that allows synchronized reconstitution of macrophage compartments by monocyte engraftment ${ }^{18,19}$.

The majority of changes in gene expression, both in fold change and numbers, occurred in the transition from monocytes to either colonic or ileal macrophages at day 4 post transfer, i.e. immediately after extravasation. Engrafted colonic and ileal macrophages segregated in gene expression according to tissue. Tissue-specific macrophage imprinting occurs hence early during development, shortly after tissue infiltration. Interestingly, two samples of engrafted ileal macrophages clustered with the colonic samples rather than the ileal ones, suggesting that the colonic signature is default. Colonic macrophages at day 12 post engraftment were also more distinct from endogenous colonic macrophages, compared to their ileal counterparts, which implies that the mature colonic gene signature might take longer time to develop or could be more heterogeneous. Recent studies have noted heterogeneity within murine blood monocytes, in particular with respect to an intermediate between the Ly6C+ and Ly6- populations ${ }^{7}$. While we currently cannot formally rule out that, colonic and ileal macrophages could hence derive from distinct precursors, we consider this however unlikely. 
Mowat and colleagues have reported a transcriptome analysis of monocytes and colonic macrophages, including intermediates of the 'waterfall' ${ }^{23}$. The authors highlighted the critical role of TGFb in the differentiation process. While a comparison of these data to ours confirmed the late onset of genes that characterize long-lived gut macrophages, the use of the distinct platforms and distinct experimental set up precluded further direct alignment. Of note, monocyte-derived cells are in our system synchronized with respect to development and therefore allow additional temporal resolution, especially with respect to final population of the scheme (P4), which comprises in the cited study ${ }^{23}$ a heterogeneous conglomerate.

With their extended half-life, Ly6C- monocytes that patrol the endothelium, have been proposed to represent vasculature-resident macrophages ${ }^{3}$. Indeed, these cells shared gene signatures with the gut resident macrophages, such as the reduction in IRF TFs following monocyte differentiation and induction of characteristic macrophage genes, such as PPARg and TGFbR. However, the comparison of these blood-resident cells to gut tissue-resident macrophages revealed also considerable differences likely associated with the residence in vasculature and the solid tissue, respectively.

Though anatomically close, the small and large intestine represent very distinct tissues, including structural dissimilarities, such as the extended ileal villi and Peyer's Patches, characteristic distinct abundance of immune cells, as well as different luminal microbiome content ${ }^{15}$. Highlighting these differences, ileum and colon display also unique susceptibility to perturbations, as for instance to a IL10R deficiency ${ }^{17,25}$. Our comparative analysis of colonic and ileal macrophages, including their generation from monocytes, might provide critical insights into the mechanism underlying segment-specific pathology resistance or susceptibility in the gut. Together with earlier reports ${ }^{7,23}$, our data sets can provide a starting point for hypothesis-driven experiments.

To conclude, we characterized here monocyte-derived intestinal macrophages generated under conditions avoiding overt inflammation. We highlight specific genes and TFs which are regulated following monocyte differentiation to generic or segment-specific intestinal macrophages. By comparing transcriptomes of early intestinal macrophages and blood-resident Ly6 $\mathrm{C}^{-}$cells, we show that the populations which share a common ancestor the $\mathrm{Ly}_{6 \mathrm{C}} \mathrm{C}^{+}$blood monocytes - show considerable overlap in gene expression, while they also display adaptation to their specific environments. Our data provide a gateway and reference point to further studies on monocyte differentiation to macrophages.

\section{Material and Methods}

\section{Mice}


Mice were kept in a specific-pathogen-free (SPF), temperature-controlled $\left(22^{\circ} \mathrm{C} \pm 1^{\circ} \mathrm{C}\right)$ facility on a reverse 12-hour light/dark cycle at the Weizmann Institute of Science. Food and water were given ad libitum. Mice were fed regular chow diet (Harlan Biotech Israel Ltd, Rehovot, Israel). The following mice strains all on C57BL6 background were used: Cx3cr $1^{\text {gfp/+ }}$ mice ${ }^{20}$, CD11c-DTR transgenic mice (B6.FVB-Tg [Itgax-DTR/GFP] 57Lan/J) ${ }^{43}$ and CX3CR1-DTR transgenic mice ${ }^{44}$. BM chimeras were generated by engraftment of 7-10 weeks old recipient mice that were irradiated the day before with a single dose of $950 \mathrm{cGy}$ using a XRAD 320 machine (Precision X-Ray (PXI). Femurs and tibiae of donor mice were removed and BM was flushed with cold PBS. BM was washed with cold PBS twice and filtered by $100 \mu \mathrm{m}$ filter. BM cells were suspended in PBS and $5 \times 10^{6}$ cells were injected IV into irradiated recipient. Mice were handled and experiments were performed under protocols approved by the Weizmann Institute Animal Care Committee (IACUC) in accordance with international guidelines.

\section{Isolation of BM monocyte grafts and monocyte transfers}

Femurs and tibias of donor mice were removed and BM was flushed with cold PBS. BM was washed with cold PBS twice and filtered by $100 \mu \mathrm{m}$ filter. Cells were suspended with PBS and loaded on equal amount of Ficoll (GE healthcare). Tubes were centrifuged $920 \mathrm{~g}$ in room temperature for 20 minutes without breaks and Buffy coats were collected and washed with cold PBS. CD11c-DTR > wt]. Cells were stained and sorted according to the following markers: CD117 ${ }^{-}$CD $11 \mathrm{~b}^{+}$CD $115^{+} \mathrm{Ly}_{6 C^{+}}$GFPint. BM chimeras were treated with $18 \mathrm{ng} / \mathrm{gram}$ bodyweight Diphtheria toxin (DTx) (Sigma-Aldrich, Cat \# D0564) for 2 consecutive days before transfer. At the day of transfer mice were injected with $10^{6} \mathrm{BM}$ monocytes IV. At days 1, 3, 5, 7 and 9 after transfer mice were injected with $9 \mathrm{ng} / \mathrm{gr}$ bodyweight DTx.

\section{Isolation of intestinal lamina propria cells}

Intestines were removed and fecal content flushed out with PBS; tubes were opened longitudinally and cut into $0.5 \mathrm{~cm}$ sections. Pieces were placed in $5 \mathrm{ml} /$ sample (up to $300 \mathrm{gr}$ of tissue) of Hanks' Balanced Salt Solution (HBSS) with 10\% heat-inactivated FCS/FBS, 2.5mM EDTA and 1 mM DL-Dithiothreitol ((DTT), Sigma-Aldrich Cat\# D9779) and incubated on a $37^{\circ} \mathrm{C}$ shaker at $300 \mathrm{rpm}$ for 30 minutes to remove mucus and epithelial cells. Following incubation, samples were vortexed for 10 seconds and filtered through a crude cell strainer. Pieces that did not pass the strainer were collected and transferred to $5 \mathrm{ml} /$ sample of PBS +/+ with 5\% heat-inactivated FCS/FBS, $1 \mathrm{mg} / \mathrm{ml}$ Collagenase VIII (Sigma-Aldrich Cat\# C2139 ) and $0.1 \mathrm{mg} / \mathrm{ml}$ DNase I (Roche Cat\# 10104159001 ). Tissue was incubated in a $37^{\circ} \mathrm{C}$ shaker at $300 \mathrm{rpm}$ for 40 minutes (colon) or 20 minutes (ileum) in the solution. After incubation, samples were vortexed for 30 seconds until tissue was dissolved, then filtered 
through a crude cell strainer. The strainer was washed with PBS -/- and centrifuged in $4{ }^{\circ} \mathrm{C}$, $375 G$ for 6 minutes. Cells were stained and subjected to FACS analysis or sorting.

\section{Isolation of murine blood monocytes}

Blood was retrieved from the vena cava, immediately placed in 150U/ml Heparin and loaded on Ficoll (GE healthcare). Tubes were centrifuged $920 \mathrm{~g}$ in room temperature for 20 minutes without breaks and Buffy coats were collected and washed with cold PBS. Cells were sorted according to the following parameters: $\mathrm{CD} 45^{+} \mathrm{CD} 11 \mathrm{~b}^{+} \mathrm{CD} 115^{+} \mathrm{Ly}_{6 \mathrm{C}^{+/}}$.

\section{Flow cytometry analysis}

Samples were suspended and incubated in staining medium (PBS without calcium and magnesium with $2 \%$ heat-inactivated Fetal Calf/Bovine Serum (FCS/FBS) and $1 \mathrm{mM}$ EDTA) containing fluorescent antibodies. Following incubation, cells were washed with staining buffer only or staining buffer with DAPI, centrifuged, filtered through $80 \mu \mathrm{m}$ filter and read. For FACS analysis, LSRFortessa (BD Biosciences) was used. For cell sorting, FACSAria III or FACSAria Fusion (BD Biosciences) were used. Results were analyzed in FlowJo software (Tree Star). Staining antibodies (clones indicated within brackets): anti-CD45 (30-F11), CD11b (M1/70), CD115/CSF-1R (AF598), Ly-6C (HK1.4), CD64/FcyRI (X54-5/7.1), CD11c (N418), anti-I-A ${ }^{b}$ (MHCII) (AF6-120.1), DAPI.

\section{RNA sequencing and analysis}

RNA-seq of populations was performed as described previously ${ }^{44}{ }^{21}$. Cells were sorted into $100 \mu$ l of lysis/binding buffer (Life Technologies) and stored at $80^{\circ} \mathrm{C}$. mRNA was captured using Dynabeads oligo(dT) (Life Technologies) according to manufacturer's guidelines. A derivation of MARS-seq ${ }^{21}$ was used to prepare libraries for RNA-seq, as detailed in Shemer et al. ${ }^{45}$. RNA-seq libraries were sequenced using the Illumina NextSeq 500. Raw reads were mapped to the genome (NCBI37/mm9) using hisat (version 0.1.6). Only reads with unique mapping were considered for further analysis. Gene expression levels were calculated and normalized using the HOMER software package (analyzeRepeats.pl rna mm9 -d<tagDir>count exons -condenseGenes -strand + -raw). Gene expression matrix was clustered using k-means algorithm (MATLAB function kmeans) with correlation as the distance metric. PCA was performed by MATLAB function pca. Gene ontology was performed by DAVID (https://david.ncifcrf.gov). Data on molecules and pathways was partly obtained by Ingenuity Pathway Analysis (IPA), Ingenuity Target Explorer, Qiagen and Metascape Pathway analysis 23.

\section{Statistics}


Results are presented as mean \pm SEM. Statistical analysis was performed using Student's $t$ test. ${ }^{*} \mathrm{P}$-value $<0.05^{* *} \mathrm{P}$-value $<0.01{ }^{* * *} \mathrm{P}$-value $<0.001$

\section{Acknowledgments}

The authors declare no competing financial interests. We would like to thank all members of the Jung laboratory for helpful discussion. We further thank the staff of the Weizmann Animal facility and the members of the FACS facility for expert advice.

\section{Funding}

Work in the Jung laboratory was supported by the European Research Council (AdvERC grant 340345), and a research grant from Roland N. Karlen Foundation.

\section{Author contributions}

M.G-V. and S.J. conceived the project and designed the experiments; S. T. helped with the manuscript preparation and bioinformatic analysis; B.B and C.T advised and assisted in adoptive transfers; T-M.S assisted in cell sorting; G.S. and D. L. performd bioinformatic analysis; E.D performed initial data analysis; S.B-H advised on data analysis; L. C-M. performed RNAseq; M.G-V. and S.J. wrote the paper; S.J. supervised the project.

\section{Data and materials availability}

The data for this study have been deposited in the database dbGAP. The sequence of XXX can be found as GenBank XXXXXX.

\section{References}

1. Ginhoux F, Guilliams M. Tissue-Resident Macrophage Ontogeny and Homeostasis. Immunity. 2016;44(3):439-449.

2. Varol C, Mildner A, Jung S. Macrophages: development and tissue specialization. Annu. Rev. Immunol. 2015;33:643-675.

3. Ginhoux F, Jung S. Monocytes and macrophages: developmental pathways and tissue homeostasis. Nat Rev Immunol. 2014;14(6):392-404.

4. Mildner A, Marinković G, Jung S. Murine Monocytes: Origins, Subsets, Fates, and Functions. Microbiol Spectr. 2016;4(5):.

5. Yona S, Kim K-W, Wolf Y, et al. Fate mapping reveals origins and dynamics of monocytes and tissue macrophages under homeostasis. Immunity. 2013;38(1):79-91.

6. Auffray C, Fogg D, Garfa M, et al. Monitoring of blood vessels and tissues by a population of monocytes with patrolling behavior. Science. 2007;317(5838):666-670.

7. Mildner A, Schönheit J, Giladi A, et al. Genomic Characterization of Murine Monocytes Reveals C/EBP\&beta; Transcription Factor Dependence of Ly6C- Cells. Immunity. 2017;46(5):849-862.e7.

8. Bain CC, Mowat AM. Intestinal macrophages - specialised adaptation to a unique environment. Eur. J. Immunol. 2011;41(9):2494-2498.

9. Zigmond E, Jung S. Intestinal macrophages: well educated exceptions from the rule. Trends in Immunology. 2013;34(4):162-168. 
10. Shaw TN, Houston SA, Wemyss K, et al. Tissue-resident macrophages in the intestine are long lived and defined by Tim-4 and CD4 expression. J. Exp. Med.

2018;7:jem.20180019-12.

11. De Schepper S, Verheijden S, Aguilera-Lizarraga J, et al. Self-Maintaining Gut Macrophages Are Essential for Intestinal Homeostasis. Cell. 2018;1-30.

12. Bujko A, Atlasy N, Landsverk OJB, et al. Transcriptional and functional profiling defines human small intestinal macrophage subsets. Journal of Experimental Medicine. 2018;215(2):441-458.

13. Tamoutounour S, Henri S, Lelouard $\mathrm{H}$, et al. CD64 distinguishes macrophages from dendritic cells in the gut and reveals the Th1-inducing role of mesenteric lymph node macrophages during colitis. Eur. J. Immunol. 2012;42(12):3150-3166.

14. Amit I, Winter DR, Jung S. The role of the local environment and epigenetics in shaping macrophage identity and their effect on tissue homeostasis. 2016;17(1):1825.

15. Mowat AM, Agace WW. Regional specialization within the intestinal immune system. Nat Rev Immunol. 2014;14(10):667-685.

16. Glocker E-O, Kotlarz D, Boztug K, et al. Inflammatory Bowel Disease and Mutations Affecting the Interleukin-10 Receptor. N. Engl. J. Med. 2009;361(21):2033-2045.

17. Zigmond E, Bernshtein B, Friedlander G, et al. Macrophage-Restricted Interleukin-10 Receptor Deficiency, but Not IL-10 Deficiency, Causes Severe Spontaneous Colitis. Immunity. 2014;40(5):720-733.

18. Varol C, Landsman L, Fogg DK, et al. Monocytes give rise to mucosal, but not splenic, conventional dendritic cells. J. Exp. Med. 2007;204(1):171-180.

19. Varol C, Vallon-Eberhard A, Elinav E, et al. Intestinal lamina propria dendritic cell subsets have different origin and functions. Immunity. 2009;31(3):502-512.

20. Jung S, Aliberti J, Graemmel P, et al. Analysis of fractalkine receptor CX(3)CR1 function by targeted deletion and green fluorescent protein reporter gene insertion. Molecular and Cellular Biology. 2000;20(11):4106-4114.

21. Jaitin DA, Kenigsberg E, Keren-Shaul H, et al. Massively parallel single-cell RNA-seq for marker-free decomposition of tissues into cell types. Science.

2014;343(6172):776-779.

22. Okayasu I, Hatakeyama S, Yamada M, et al. A novel method in the induction of reliable experimental acute and chronic ulcerative colitis in mice. YGAST. 1990;98(3):694-702.

23. Zhou Y, Bin Zhou, Pache L, et al. Metascape provides a biologist-oriented resource for the analysis of systems-level datasets. Nat Comms. 2019;1-10.

24. Schridde A, Bain CC, Mayer JU, et al. Tissue-specific differentiation of colonic macrophages requires TGF $\beta$ receptor-mediated signaling. Mucosal Immunol. 2017;10(6):1387-1399.

25. Bernshtein $B$, Curato $C$, loannou $M$, et al. IL-23-producing IL-10Ra-deficient gut macrophages elicit an IL-22-driven proinflammatory epithelial cell response. Sci Immunol. 2019;4(36):eaau6571.

26. Foey AD, Parry SL, Williams LM, et al. Regulation of monocyte IL-10 synthesis by endogenous IL-1 and TNF-alpha: role of the p38 and p42/44 mitogen-activated protein kinases. J. Immunol. 1998;160(2):920-928.

27. Houle M, Thivierge M, Le Gouill C, Stankovà J, Rola-Pleszczynski M. IL-10 upregulates CCR5 gene expression in human monocytes. Inflammation. 1999;23(3):241-251.

28. Cassatella MA, Gasperini S, Bovolenta C, et al. Interleukin-10 (IL-10) selectively enhances CIS3/SOCS3 mRNA expression in human neutrophils: evidence for an IL10 -induced pathway that is independent of STAT protein activation. Blood. 1999;94(8):2880-2889.

29. Diehl GE, Longman RS, Zhang J-X, et al. Microbiota restricts trafficking of bacteria to mesenteric lymph nodes by CX(3)CR1(hi) cells. Nature. 2013;494(7435):116-120. 
30. Aychek T, Mildner A, Yona S, et al. IL-23-mediated mononuclear phagocyte crosstalk protects mice from Citrobacter rodentium-induced colon immunopathology. Nat Comms. 2015;6(1):6525.

31. Masuda K, Ripley B, Nyati KK, et al. Arid5a regulates naive CD4+ T cell fate through selective stabilization of Stat3 mRNA. Journal of Experimental Medicine. 2016;213(4):605-619.

32. Tsoyi K, Geldart AM, Christou H, et al. Elk-3 is a KLF4-regulated gene that modulates the phagocytosis of bacteria by macrophages. Journal of Leukocyte Biology. 2015;97(1):171-180.

33. Ousingsawat $\mathrm{J}$, Wanitchakool $\mathrm{P}, \mathrm{Kmit} \mathrm{A}$, et al. Anoctamin 6 mediates effects essential for innate immunity downstream of P2X7 receptors in macrophages. Nat Comms. 2015;6(1):6245.

34. Khouri R, Báfica A, Silva MDPP, et al. IFN-beta impairs superoxide-dependent parasite killing in human macrophages: evidence for a deleterious role of SOD1 in cutaneous leishmaniasis. The Journal of Immunology. 2009;182(4):2525-2531.

35. Zhong S, Li L, Zhang Y-L, et al. Acetaldehyde dehydrogenase 2 interactions with LDLR and AMPK regulate foam cell formation. J. Clin. Invest. 2019;129(1):252-267.

36. Barneda-Zahonero $\mathrm{B}$, Román-González L, Collazo $\mathrm{O}$, et al. HDAC7 is a repressor of myeloid genes whose downregulation is required for transdifferentiation of pre-B cells into macrophages. PLoS Genet. 2013;9(5):e1003503.

37. Harris ES, Gauvin TJ, Heimsath EG, Higgs HN. Assembly of filopodia by the formin FRL2 (FMNL3). Cytoskeleton (Hoboken). 2010;67(12):755-772.

38. Rosas M, Davies LC, Giles PJ, et al. The transcription factor Gata6 links tissue macrophage phenotype and proliferative renewal. Science. 2014;344(6184):645-648.

39. Yamasaki R, Lu H, Butovsky O, et al. Differential roles of microglia and monocytes in the inflamed central nervous system. Journal of Experimental Medicine. 2014;211(8):1533-1549.

40. Rivollier A, He J, Kole A, Valatas V, Kelsall BL. Inflammation switches the differentiation program of Ly6Chi monocytes from antiinflammatory macrophages to inflammatory dendritic cells in the colon. Journal of Experimental Medicine. 2012;209(1):139-155.

41. Zigmond E, Varol C, Farache J, et al. Ly6Chi Monocytes in the Inflamed Colon Give Rise to Proinflammatory Effector Cells and Migratory Antigen-Presenting Cells. Immunity. 2012;37(6):1076-1090.

42. Avraham-Davidi I, Yona S, Grunewald M, et al. On-site education of VEGF-recruited monocytes improves their performance as angiogenic and arteriogenic accessory cells. Journal of Experimental Medicine. 2013;210(12):2611-2625.

43. Jung $S$, Unutmatz D, Wong $P$, et al. In Vivo Depletion of CD11c Dendritic Cells Abrogates Priming of CD8 T Cells by Exogenous Cell-Associated Antigens. Immunity. 2002;17:211-220.

44. Diehl GE, Longman RS, Zhang J-X, et al. Microbiota restricts trafficking of bacteria to mesenteric lymph nodes by $\mathrm{CX}_{3} \mathrm{CR} 1^{\text {hi }}$ cells. Nature. (2013) 494(7435):116-120.

45. Lavin $Y$, Winter D, Blecher-Gonen R, et al. Tissue-Resident Macrophage Enhancer Landscapes Are Shaped by the Local Microenvironment. Cell. 2014;159(6):13121326.

46. Shemer A, Grozovski J, Tay TL, et al. Engrafted parenchymal brain macrophages differ from microglia in transcriptome, chromatin landscape and response to challenge. Nat Comms. 2018;1-16. 


\section{Figure Legends}

Figure 1. Transcriptome analysis of $\mathrm{CX}_{3} \mathrm{CR} 1^{+}$monocyte graft - derived colonic and ileal macrophages isolated from macrophage depleted animals

A) Experimental protocol. Briefly, [CD11C-DTR > C57BL/6] BM chimeras were treated as indicated with DTx. $2 \times 10^{6}$ CD $117^{-}$CD $11 b^{+}$CD $115^{+}$Ly6C $^{+}$GFP int $B M$ monocytes isolated from $\mathrm{CX} 3 \mathrm{CR} 1^{\mathrm{GFP} /+}$ mice were injected intravenously. Macrophages were sorted from the colon and ileum at days 4, 8 and 12 post-transfer. Experiment was repeated 3 times, total 3-4 samples from each tissue at each time point.

B) Graft-derived macrophages were sorted at days 4, 8 and 12 post transfer based on CD45.1 and CX3CR1 (GFP) expression. GFP ${ }^{+}$cells also express CD64, CD11b and variable levels of CD11c, and lacked Ly6C expression.

C) Principal component analysis (PCA) of transcriptomes of BM monocytes and grafted cells from colon and ileum at 4, 8 and 12 days post-transfer. Analysis performed in MATLAB.

D) Expression heat map of 4213 genes that show at least 4 fold change across all samples in the dataset, divided to 7 clusters by the unbiased K-means algorithm in MATLAB. (Suppl. Table 1, data sets 1,2, 5-11)

E) Representative genes from heat map in (D).

F-H) Double volcano plots of genes that change from monocytes to day 4 , day 4 to day 8 and day 8 to day 12 in the colon (green dots) and ileum (blue dots). Blue and green squares indicate genes that are significantly (Student's T-test, $P$-value $<0.05$ ) changed between the different time points. Numbers within blue and green squares represent the number of genes in the square, namely genes that significantly change. Arrows indicate up -or down-regulation.

\section{Figure 2. Differences between acute monocyte graft -derived macrophages and resident intestinal macrophages}

A) Volcano plots of genes that change from engrafted macrophages at day 12 and resident macrophages in the colon (left) and ileum (right). Grey dots represent genes that do not significantly change (P-value>0.05), black dots represent genes that significantly change (P-value<0.05). Green and blue squares mark genes with at least 2-fold log fold change (equals 4-fold read change). Numbers inside squares indicate number of genes in square, namely genes that significantly change. Arrows indicate up- or down-regulated genes in resident macrophages compared to engrafted macrophages at day 12 post transfer.

B) Example genes characterizing long-lived intestinal macrophages according to Shaw et al.

C) Example genes highly expressed in resident macrophages compared to engrafted cells.

D) Example genes highly expressed in engrafted cells compared to long-lived macrophages. 
E) Example genes participating in IL10 / IL10R axis.

Figure 3. Changes in gene expression during conversion of monocytes into colonic or ileal macrophages

A) Example genes that are significantly up-regulated in intestinal macrophages from monocytes to day 4: either up-regulated in the colon but do not change in the ileum, or significantly up-regulated in the ileum but do not change in the colon. Numbers indicate number of genes to follow the trend. Only genes which have significantly different levels between colonic and ileal macrophages at all time points (monocytes > day 4, day4 > day8, day8 > day12) were selected.

B) Example genes that are significantly down-regulated from monocytes to day 4 in intestinal macrophages: either down-regulated in the colon but do not change in the ileum, or significantly down-regulated in the ileum but do not change in the colon. Numbers indicate number of genes to follow the trend.

C) Example genes that are either up-regulated in the colon from monocytes to day 4 and down-regulated in the ileum from monocytes to day 4 or vice versa.

\section{Figure 4. Changes in gene expression during conversion of monocytes into generic} intestinal macrophages

A) Dot plot for genes whose expression changes at least 2-fold between both monocytes to engrafted colonic macrophages at day 4 and monocytes to engrafted ileal macrophages at day 4. Black dots: genes that significantly change in the transition to both tissues. Blue dots: genes that significantly change in monocytes to engrafted ileum macrophages only. Green dots: genes that significantly change from monocytes to engrafted colon macrophages only. Only genes that significantly changed from monocytes to both colonic and ileal macrophages at day 4 , but expression levels not distinct between colon and ileum at day 4 , were selected.

B) Log averages of all genes that are up-regulated (left) or down-regulated (right) in generic intestinal macrophages compared to monocytes. Red line marks threshold (50 reads, 5.672 in $\log _{2}$ ) of very low/no expression levels.

C) Top 5 GO pathways that are related to genes that are 'selectively expressed in generic intestinal macrophages compared with monocytes (left) or selectively expressed in monocytes compared with macrophages (right). Bars show percent of genes (out of total genes) that are related to each GO term, red markers illustrate the p-value of each GO term.

D) Example genes from GO pathways. Bars indicate mean normalized reads, error bars represent SEM. 
Figure 5. Transcription factors which change during intestinal macrophage differentiation

A) Expression graphs of 3 TF families: Cebp (left), E2f (middle) and Egr (right). Lines mark average normalized reads, error bars represent SEM.

B) Representative TFs that are down-regulated in generic intestinal macrophages.

C) Representative TFs that are up-regulated in generic intestinal macrophages.

Figure 6. Gene expression changes in $\mathrm{Ly}_{6} \mathrm{C}^{+}$monocytes after differentiating to tissueresident cells

A) Scheme of monocyte development and fates during physiology and inflammation

B) Fold change heat map of all 1303 genes that significantly change during the transition of Ly6 $\mathrm{C}^{+}$blood monocytes to Ly6C- blood monocytes and BM Ly6 $\mathrm{C}^{+}$monocytes to engrafted intestinal macrophages at day 4 . Colors represent fold change between tissue-resident cells and their respective precursor cells. (Suppl. Table 1, data sets 1, 2, 5, 12,13)

C) Representative monocyte-related genes.

D) Representative genes related to monocyte-derived cells

E) Expression of TFs of the Cebp and Irf gene families. 


\section{Supplementary Figures}

\section{Supplementary Figure 1. Comparison of gene expression in monocytes and resident} colonic and ileal macrophages.

A) Sorting schemes for BM Monocytes and resident intestinal macrophages isolated from $\mathrm{CX}_{3} \mathrm{CR} 1$-heterozygous $\mathrm{CX}_{3} \mathrm{CR} 1^{\mathrm{GFP} /+} \mathrm{C} 57 \mathrm{BL} / 6$ mice according to the following surface markers: CD117 CD11b ${ }^{+}$CD115 ${ }^{+}$Ly6C $^{\text {high }} \mathrm{CX}_{3} \mathrm{CR} 1 / \mathrm{GFP}^{\text {int }}$ (monocytes); DAPI- CD $45^{+}$ CD11 $\mathrm{b}^{+} \mathrm{CD}^{+} 4^{+} \mathrm{Ly}^{-} \mathrm{CHCII}^{+}$(macrophages).

B) Principal component analysis (PCA) of all 12346 genes expressed in BM monocytes and resident macrophages from the colon ad ileum of $\mathrm{CX}_{3} \mathrm{CR} 1^{+/ g f p}$ mice. Analysis was performed in MATLAB. BM monocytes $(n=3)$; colon, ileum macrophages ( $n=4$, each) (Suppl. Table 1, data sets 1, 2, 5).)

C) Heat map of transcriptomes and representative genes. 6200 genes that show at least 2 fold change across all samples in the dataset divided to 4 clusters by the K-means algorithm in MATLAB. Visualization performed in Gene-E. (Suppl. Table 1, data sets 1, 2, 5). GO pathway analysis of clusters.

D) Example genes for the clusters in (C).

\section{Supplementary Figure 2. Analysis of host tissue for graft-derived cells}

A) Fluorescent images of ileum of $\mathrm{CX}_{3} \mathrm{CR} 1^{\mathrm{GFP} /+}$ mouse (top) and DTx-treated [CD11C-DTR > WT] BM chimeras engrafted with $\mathrm{CX}_{3} \mathrm{CR} 1^{\mathrm{GFP} /+}$ monocytes at the indicated days posttransfer.

B) Flow cytometric analysis of engrafted (CD45.1) and host (CD45.2) intestinal macrophages in the adoptive transfer model involving monocyte engraftment of DTx-treated [CD11CDTR > WT] BM chimeras; representative analyzed sample is from day 8 post engraftment.

\section{Supplementary Figure 3. Analysis of host tissue for graft-derived cells}

A) Elbow plot to determine number of clusters. Since the plot was continuous, visual inspection was performed with different cluster numbers; according to cluster homogeneity we selected $\mathrm{k}=7$.

B) Schematic of clusters related to Fig 1D

C) Metascape pathway analysis cluster II, genes shared by colonic and ileal monocyte graftderived macrophages.

D) Metascape pathway analysis of cluster III, genes preferentially expressed by colonic monocyte graft-derived macrophages.

E) Metascape pathway analysis of cluster IV, genes preferentially expressed by ileal monocyte graft-derived macrophages. 


\section{Supplementary Figure 4. Analysis of expression of pro-inflammatory genes in adoptive transfer model and DSS-induced colitis model.}

Expression of genes in engrafted colonic and ileal macrophages, resident colonic and ileal macrophages at steady state, infiltrating monocytes and resident macrophages isolated from the colon of mice challenged with $1.5 \%$ DSS for 7 days and their respective controls.

\section{Supplementary Figure 5. Comparison of graft and engrafted cells to recent monocytic} infiltrates in the colon, identified by their Ly6C surface expression

\section{A) Representative flow cytometric analysis of colonic myeloid cells. Recent monocytic} infiltrates and macrophages in the colon were defined as $\mathrm{CD} 45^{+} \mathrm{CD} 11 \mathrm{~b}^{+} \mathrm{Ly}_{6 \mathrm{C}^{+} \mathrm{MHCIl}^{-} \text {and }}$ CD45 ${ }^{+} \mathrm{CD} 11 \mathrm{~b}^{+} \mathrm{CD} 64^{+} \mathrm{Ly}^{-} \mathrm{C}^{-} \mathrm{MHCI}^{+}$cells, respectively and sorted accordingly for RNAseq.

B) Representative correlation coefficients between indicated cell populations (Suppl. Table 1, data sets $3,4,5,6)$.

C) Representative genes differentially expressed between monocyte graft, engrafted cells (d4), early monocyte infiltrates and resident macrophages

\section{Supplementary Figure 6. Comparison of colonic macrophages generated upon monocyte transfer into [CD11C-DTR > WT] and [CX3CR1-DTR > WT] BM chimeras treated with DTx.}

A) Venn diagrams of gene lists up- and downregulated as compared to the monocyte graft.

B) Representative upregulated genes similarly expressed between the colonic macrophages as compared to the monocyte graft; engrafted cells isolated from [CD11c-DTR > WT] mice (d12); engrafted cells isolated from [CX3CR1-DTR > WT] mice (d14).

C) Representative downregulated genes similarly expressed between the colonic macrophages as compared to the monocyte graft; engrafted cells isolated from [CD11cDTR > WT] mice (d12); engrafted cells isolated from [CX3CR1-DTR > WT] mice (d14).

\section{Supplementary Figure 7. Gating strategy for sorting of Ly6C $\mathrm{C}^{+}$and Ly6C- blood}

monocytes. Representative flow cytometric analysis indicating gates used for isolation of blood monocyte subsets. 


\section{Supplementary Table 1}

List of RNAseq data sets prepared and used in this study

\begin{tabular}{|c|c|c|c|c|c|}
\hline $\begin{array}{c}\text { data set } \\
\#\end{array}$ & description & mice & $\begin{array}{l}\text { number of } \\
\text { repeats }\end{array}$ & \begin{tabular}{|c|}
$\begin{array}{c}\text { main relevant } \\
\text { figure(s) }\end{array}$ \\
\end{tabular} & comments \\
\hline 1 & Colonic macrophages & C57BL/ 6 & 4 & Suppl Figure 1 & \\
\hline 2 & Ileum macrophages & C57BL/ 6 & 4 & Suppl Figure 2 & \\
\hline 3 & Colon macrophages & C57BL/ 6 & 4 & Suppl Figure 4 & $\begin{array}{l}\text { data } 3,4 \text { are from } \\
\text { same animals }\end{array}$ \\
\hline 4 & Colonic 'monocytes' & C57BL/ 6 & 4 & Suppl Figure 4 & $\begin{array}{c}\text { data } 3,4 \text { are from } \\
\text { same animals }\end{array}$ \\
\hline 5 & BM monocytes (graft) & $\begin{array}{c}\text { C57BL/ } 6 \text { CX3CR1-GFP } \\
\text { (CD45.1) }\end{array}$ & 3 & Figure 1 & \\
\hline 6 & $\begin{array}{l}\text { Colon, Graft-derived } \\
\text { cells Day } 4\end{array}$ & $\begin{array}{c}\text { C57BL/ 6 CX3CR1-GFP } \\
\text { (CD45.1) } \\
\end{array}$ & 3 & Figure 1 & $\begin{array}{l}\text { CD11c-DTR } \\
\text { recipient }\end{array}$ \\
\hline 7 & $\begin{array}{l}\text { Colon, Graft-derived } \\
\text { cells Day } 8\end{array}$ & $\begin{array}{c}\text { C57BL/ } 6 \text { CX3CR1-GFP } \\
\text { (CD45.1) }\end{array}$ & 3 & Figure 1 & $\begin{array}{l}\text { CD11c-DTR } \\
\text { recipient }\end{array}$ \\
\hline 8 & $\begin{array}{l}\text { Colon, Graft-derived } \\
\text { cells Day } 12\end{array}$ & $\begin{array}{c}\text { C57BL/ } 6 \text { CX3CR1-GFP } \\
\text { (CD45.1) }\end{array}$ & 4 & Figure 1 & $\begin{array}{l}\text { CD11c-DTR } \\
\text { recipient }\end{array}$ \\
\hline 9 & $\begin{array}{l}\text { lleum, Graft-derived cells } \\
\text { Day } 4\end{array}$ & $\begin{array}{c}\text { C57BL/ } 6 \text { CX3CR1-GFP } \\
\text { (CD45.1) } \\
\end{array}$ & 4 & Figure 1 & $\begin{array}{l}\text { CD11c-DTR } \\
\text { recipient }\end{array}$ \\
\hline 10 & $\begin{array}{l}\text { lleum, Graft-derived cells } \\
\text { Day } 8\end{array}$ & $\begin{array}{c}\text { C57BL/ } 6 \text { CX3CR1-GFP } \\
\text { (CD45.1) }\end{array}$ & 4 & Figure 1 & $\begin{array}{l}\text { CD11c-DTR } \\
\text { recipient } \\
\end{array}$ \\
\hline 11 & $\begin{array}{l}\text { lleum, Graft-derived cells } \\
\text { Day } 12\end{array}$ & $\begin{array}{c}\text { C57BL/ 6 CX3CR1-GFP } \\
\text { (CD45.1) } \\
\end{array}$ & 4 & Figure 1 & $\begin{array}{l}\text { CD11c-DTR } \\
\text { recipient }\end{array}$ \\
\hline 12 & Blood monocyte Ly6C+ & C57BL/ 6 & 3 & Figure 6 & \\
\hline 13 & Blood monocyte Ly6C- & C57BL/ 6 & 3 & Figure 6 & \\
\hline 14 & $\begin{array}{l}\text { Colon, Graft-derived } \\
\text { cells Day } 14\end{array}$ & $\begin{array}{c}\text { C57BL/ } 6 \text { CX3CR1-GFP } \\
\text { (CD45.1) }\end{array}$ & 4 & Suppl Figure 6 & $\begin{array}{l}\text { CX3CR1-DTR } \\
\text { recipient }\end{array}$ \\
\hline
\end{tabular}


A CD11c-DTR (CD45.2)

DTx injection

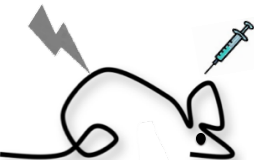

WT (CD45.2)

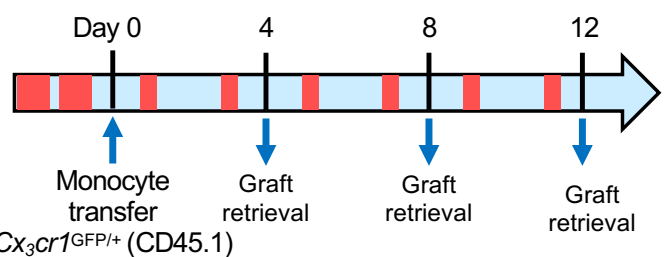

B

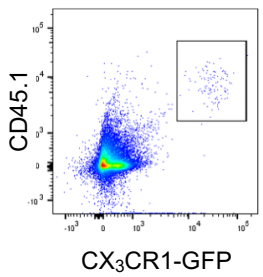
$\mathrm{Cx}_{3} \mathrm{Cr}^{\mathrm{GFP} /+}$ (CD45.1)
C

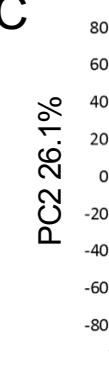

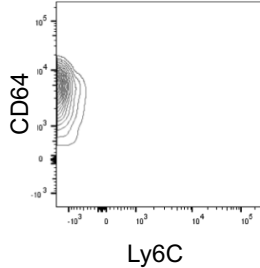

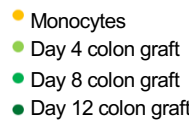

- Day 4 ileum graft

- Day 8 ileum graft - Day 12 ileum graft
D

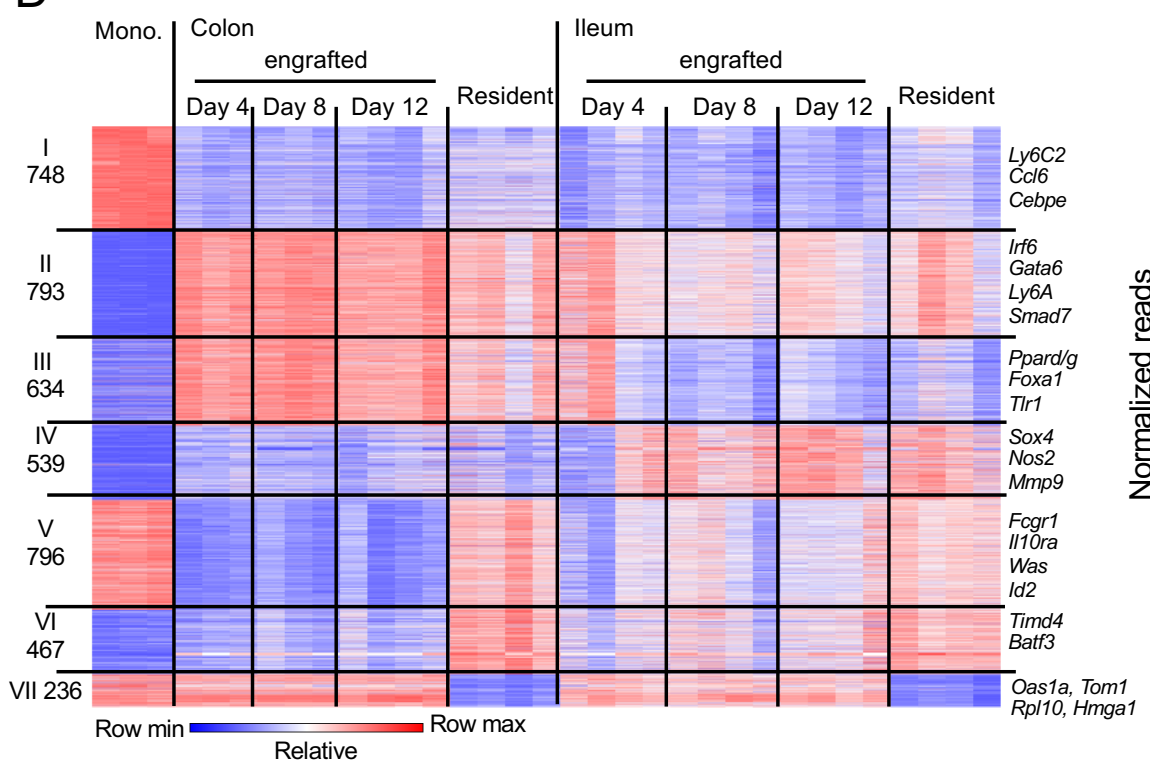

F

E
- 88

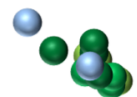

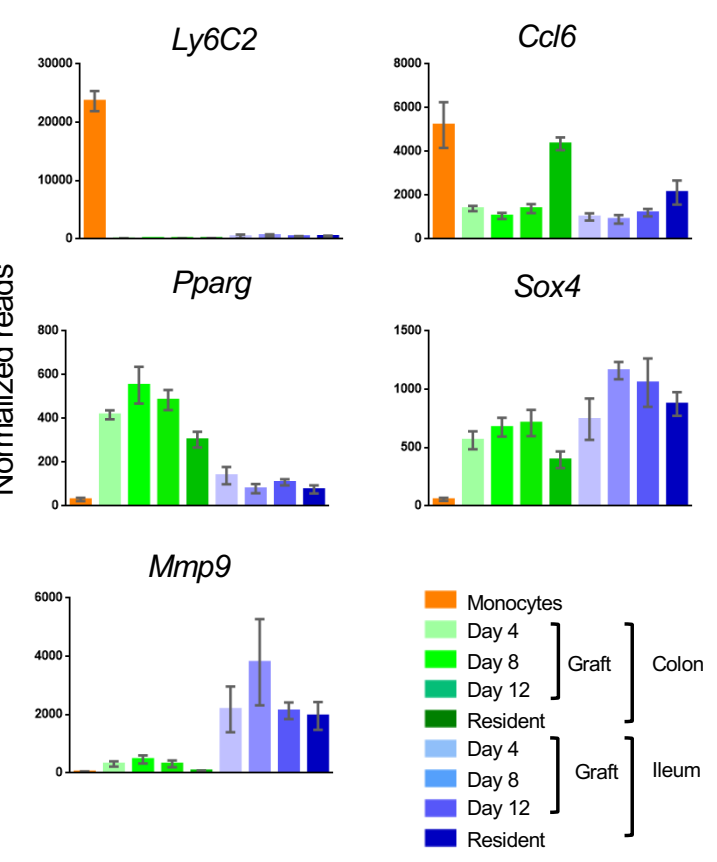

$\mathrm{H} \quad$ Engrafted macrophages

day $8 \rightarrow$ day 12
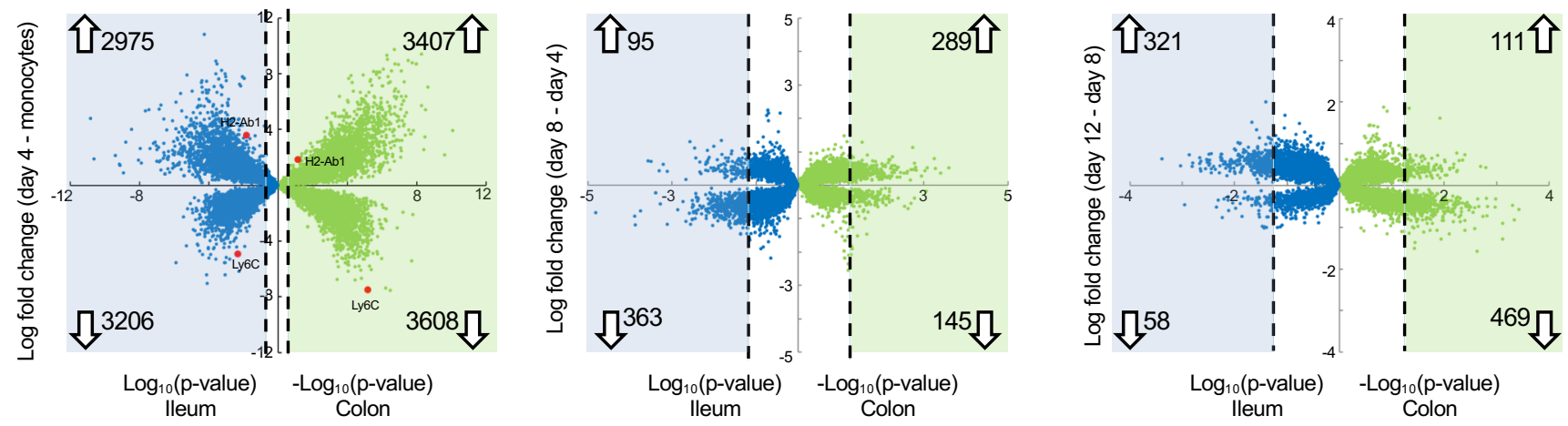

Gross-Vered et al. Fig. 1 
A

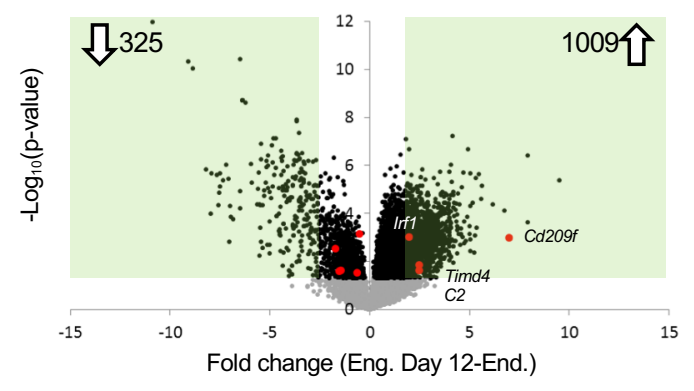

B

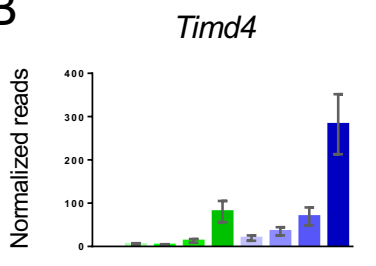

C

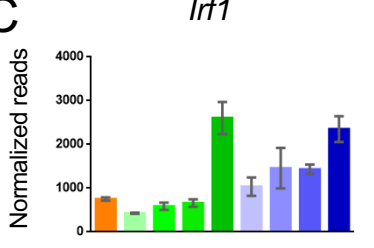

D Oas1a

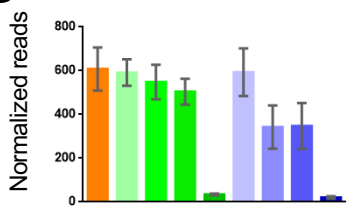

E

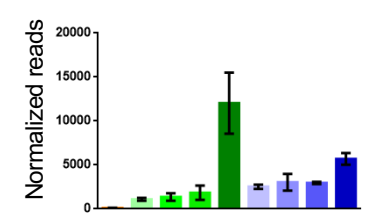

CD209f

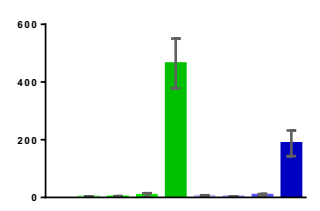

AxI

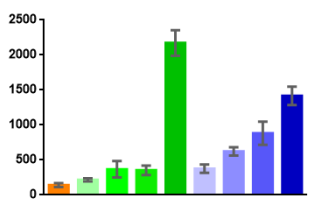

Tom1
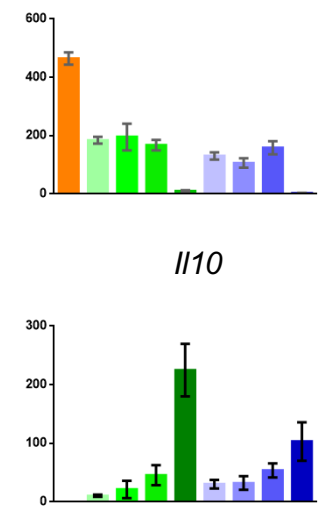

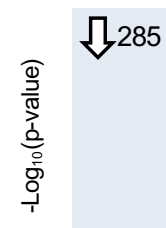

$\underline{\text { lleum }}$

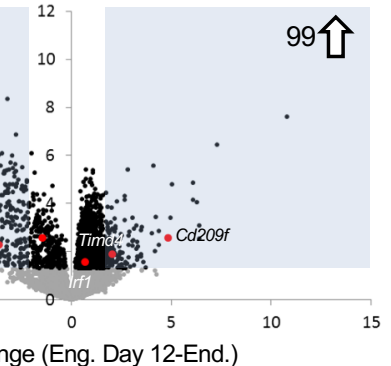

C2

Rusc2

Emp1
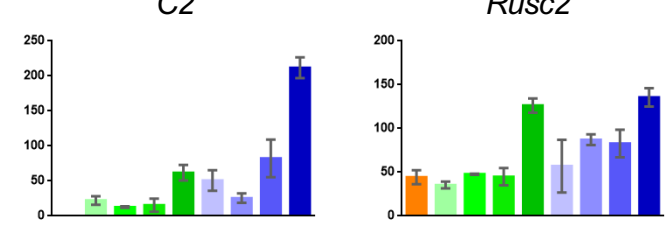

Ccl2

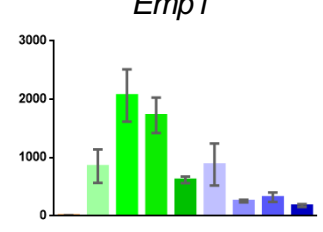

Batf3
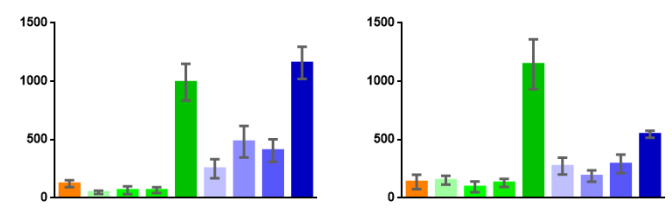

H2-Ab1

Hmga1

Rp/10
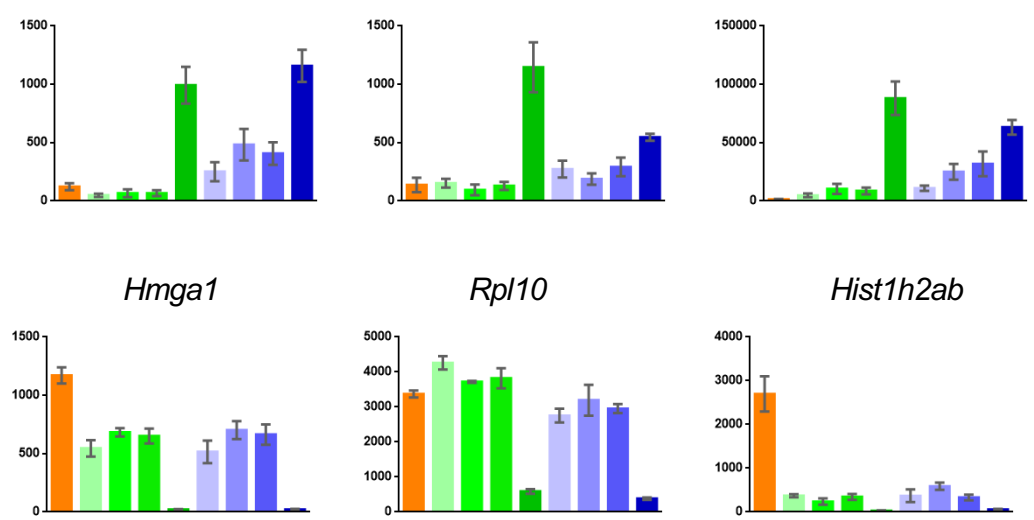

Ccr5

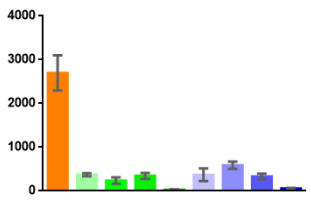

II10ra
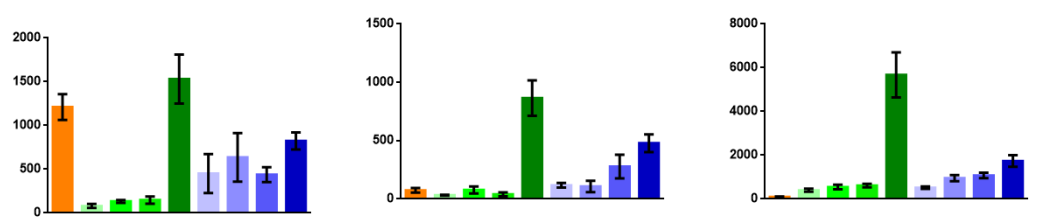

Gross-Vered et al. Fig. 2 

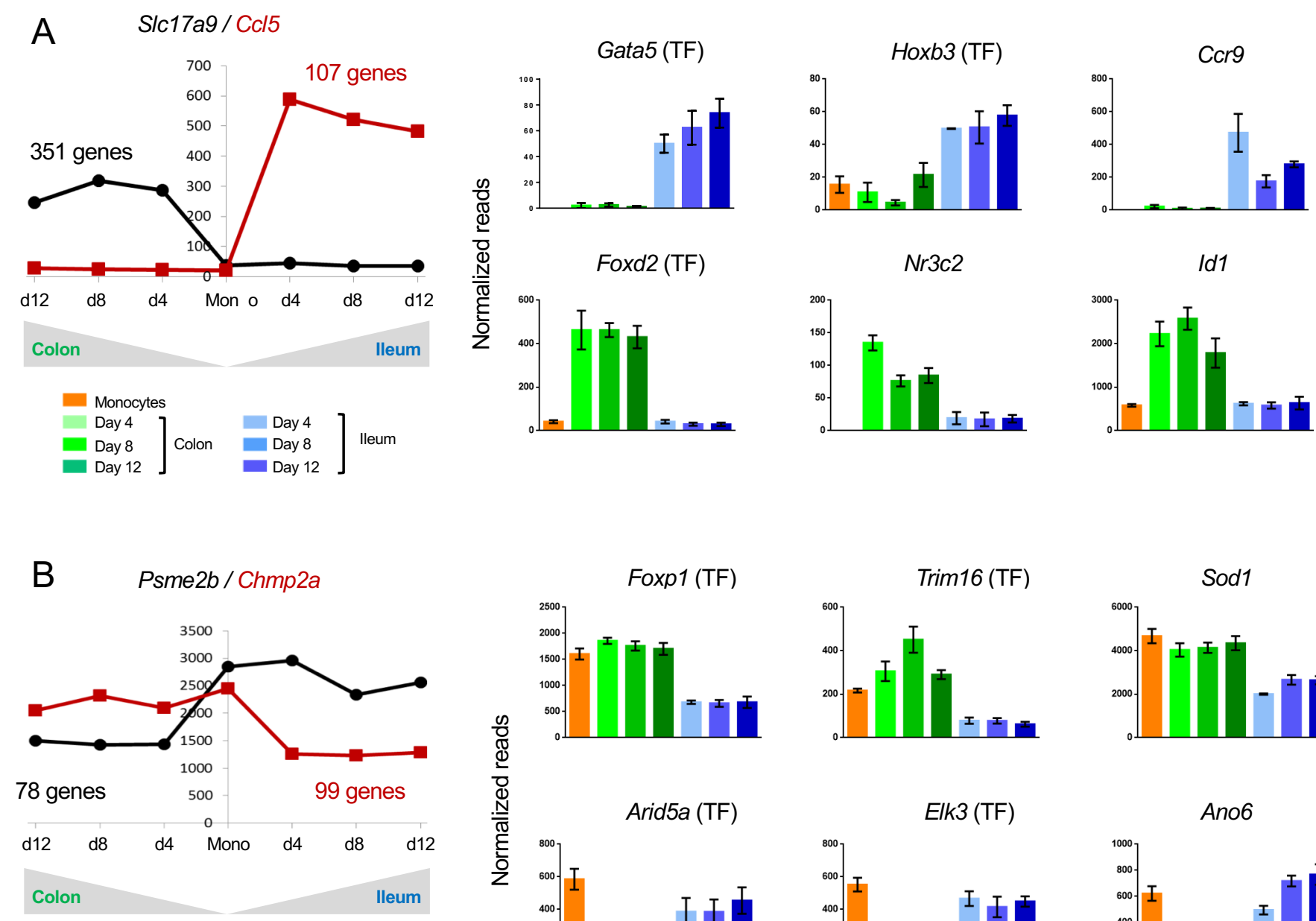

$$
\left.\left.\begin{array}{l}
\text { Monocytes } \\
\text { Day } 4 \\
\text { Day } 8 \\
\text { Day } 12
\end{array}\right] \text { Colon } \begin{array}{l}
\text { Day } 4 \\
\text { Day } 8 \\
\text { Day } 12
\end{array}\right] \text { Ileum }
$$

C Nupr1/Slc43a3
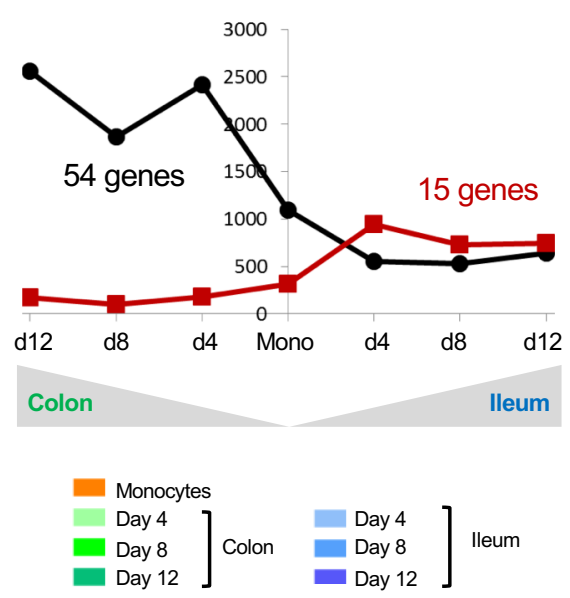

Foxp1 (TF)

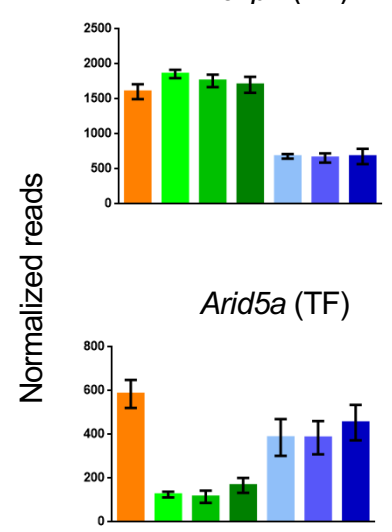

KIf4 (TF)

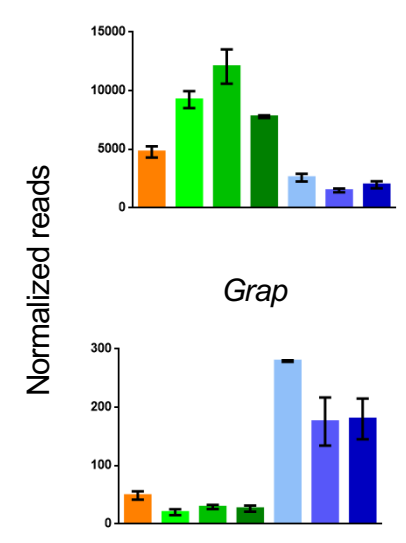

S100a6

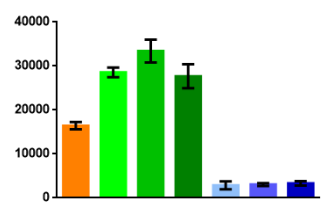

Hdac7

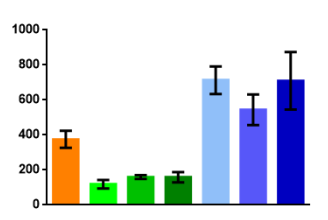

Sod1

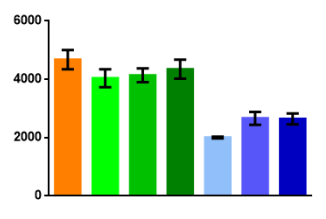

Ano6

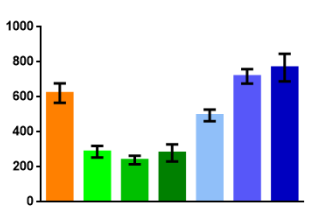

Aldh2

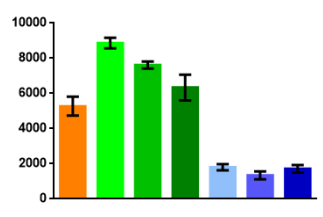

Fmnl3

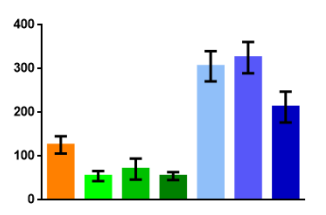




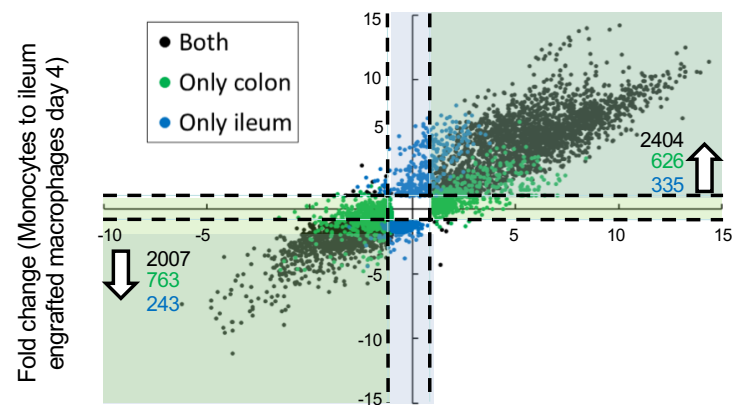

Fold change (Monocytes to Colon

C engrafted macrophages day 4)

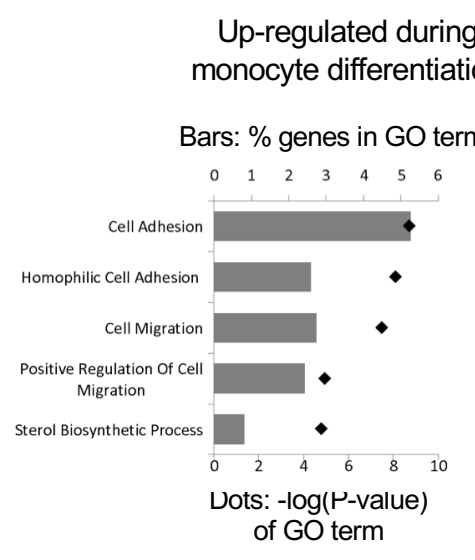

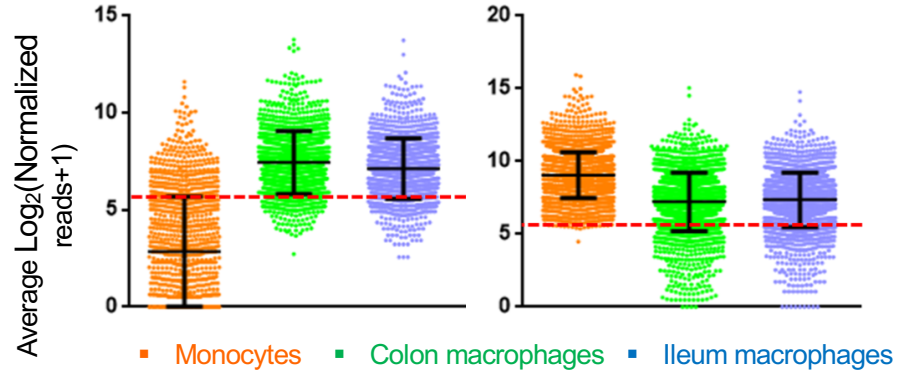

D

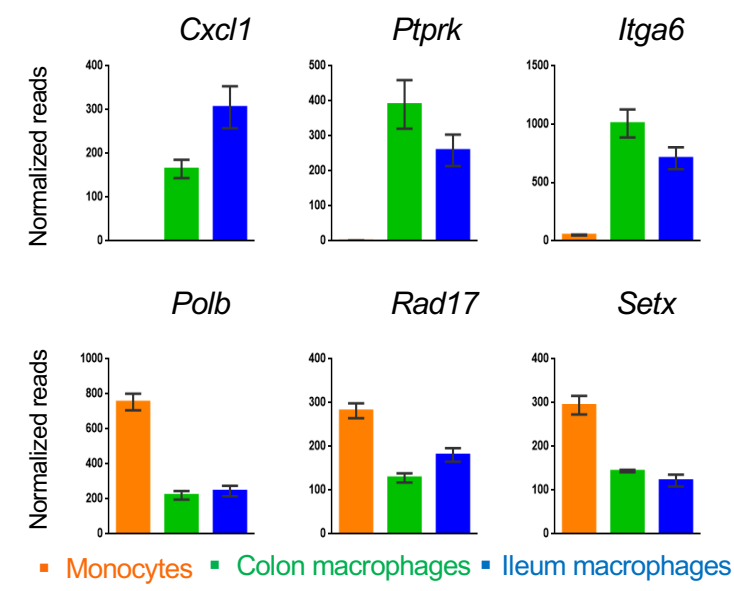

Gross-Vered et al. Fig. 4 
A
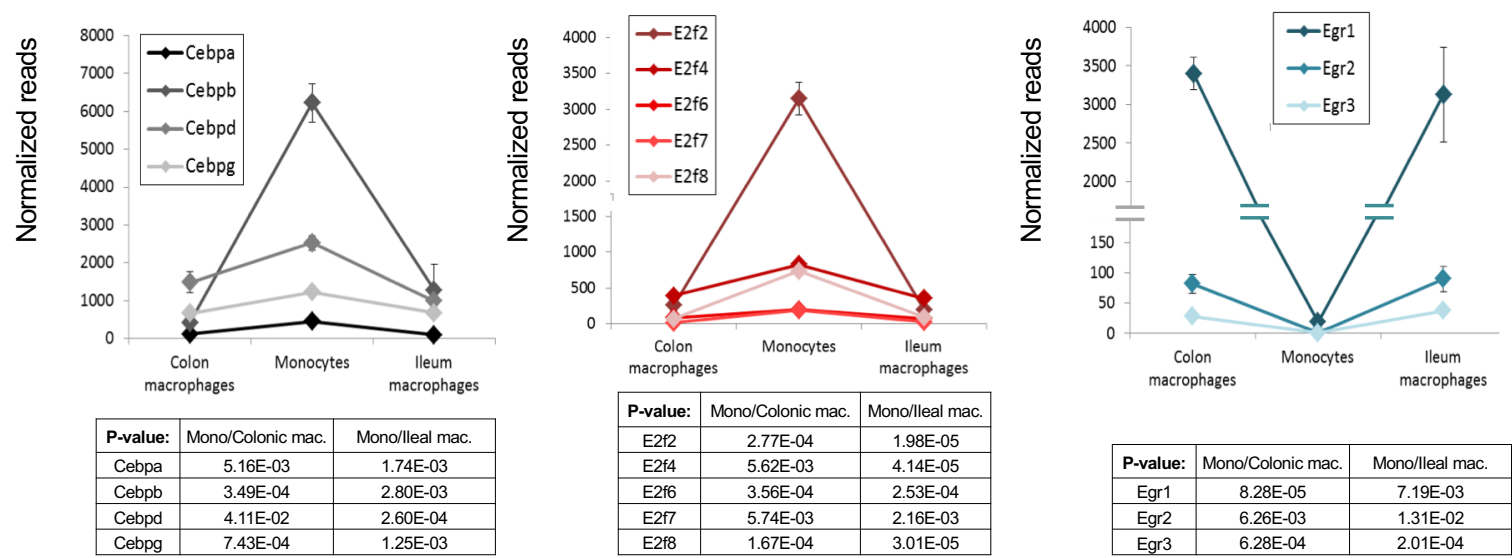

\begin{tabular}{|c|c|c|}
\hline P-value: & Mono/Colonic mac. & Mono/lleal mac. \\
\hline Egr1 & $8.28 \mathrm{E}-05$ & $7.19 \mathrm{E}-03$ \\
\hline Egr2 & $6.26 \mathrm{E}-03$ & $1.31 \mathrm{E}-02$ \\
\hline Egr3 & $6.28 \mathrm{E}-04$ & $2.01 \mathrm{E}-04$ \\
\hline
\end{tabular}

B

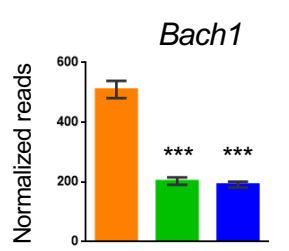

Stat3
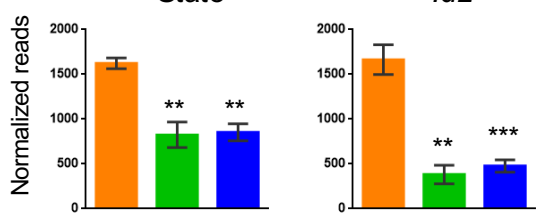

Id2
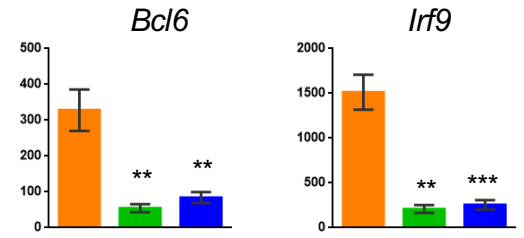

Spi1
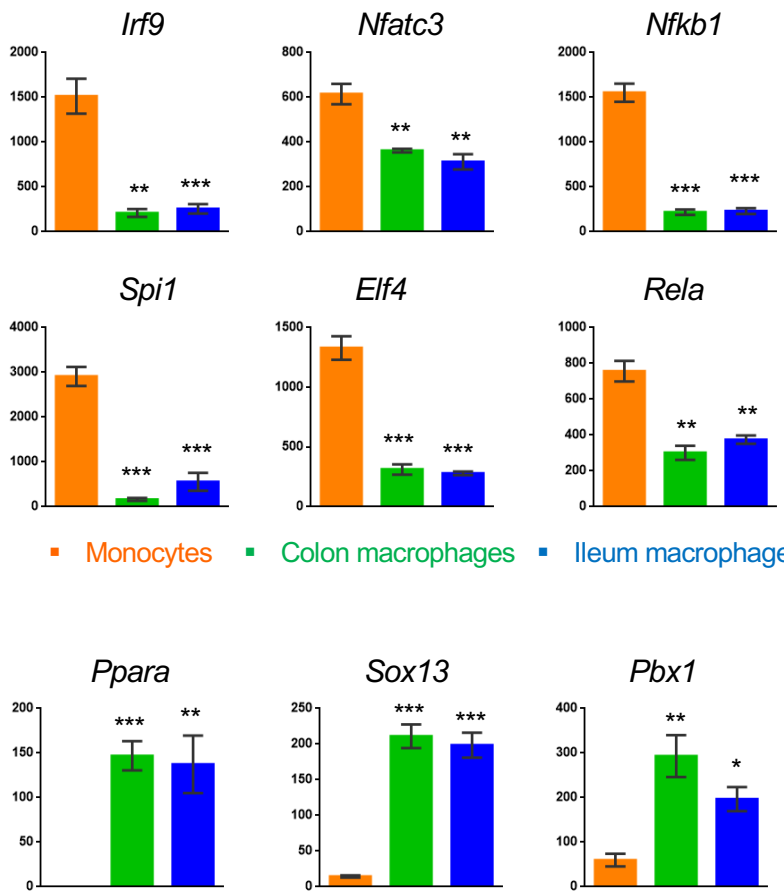

C

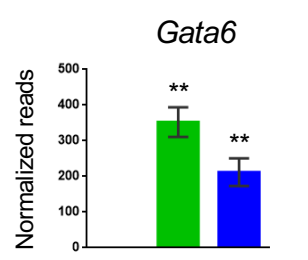

Isx

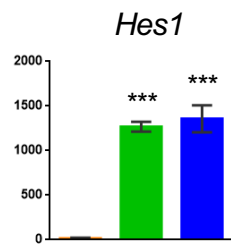

Foxa1
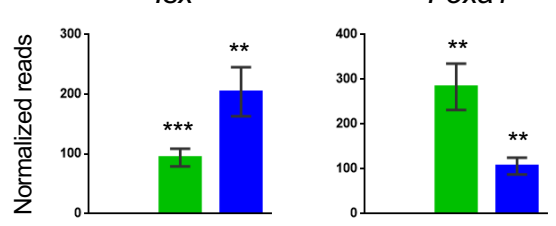

Hoxa5

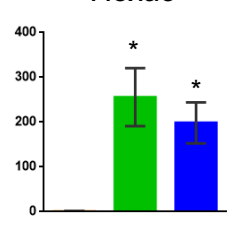

- Monocytes - Colon macrophages - lleum macrophages

Gross-Vered et al. Fig. 5 
A
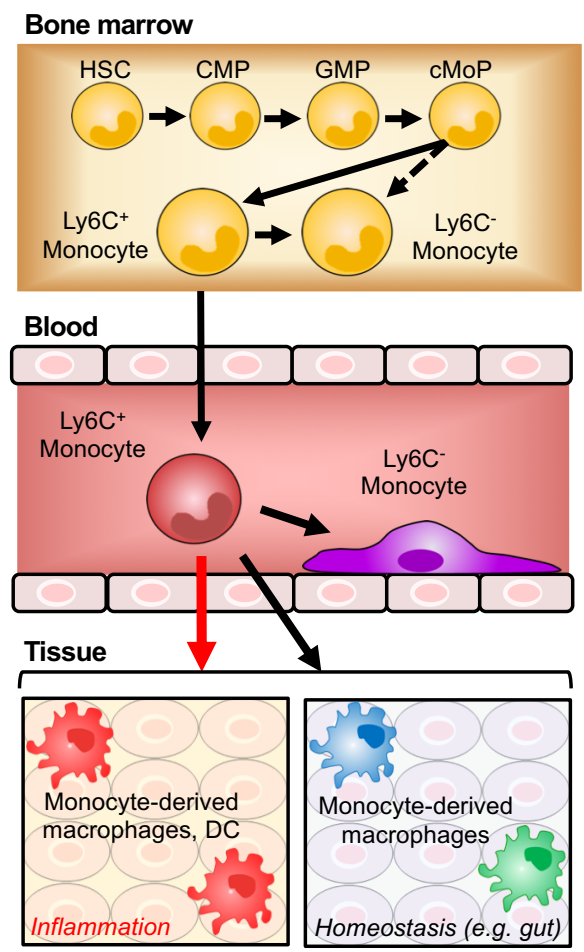

C

$$
\text { C Ly6c2 }
$$
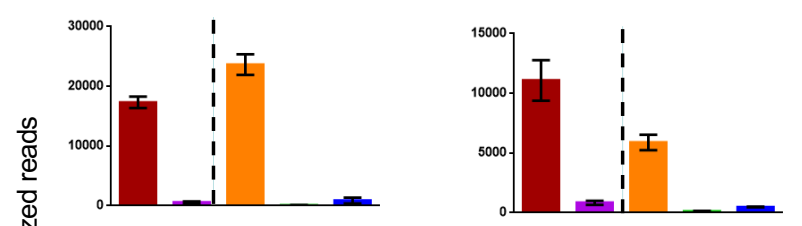

Myd88

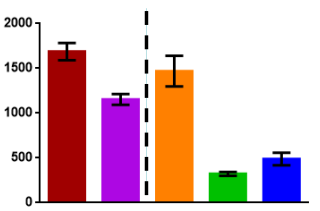

Mmp8

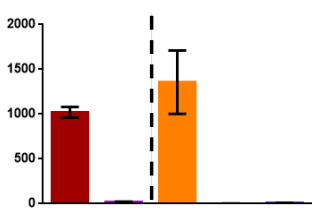

B

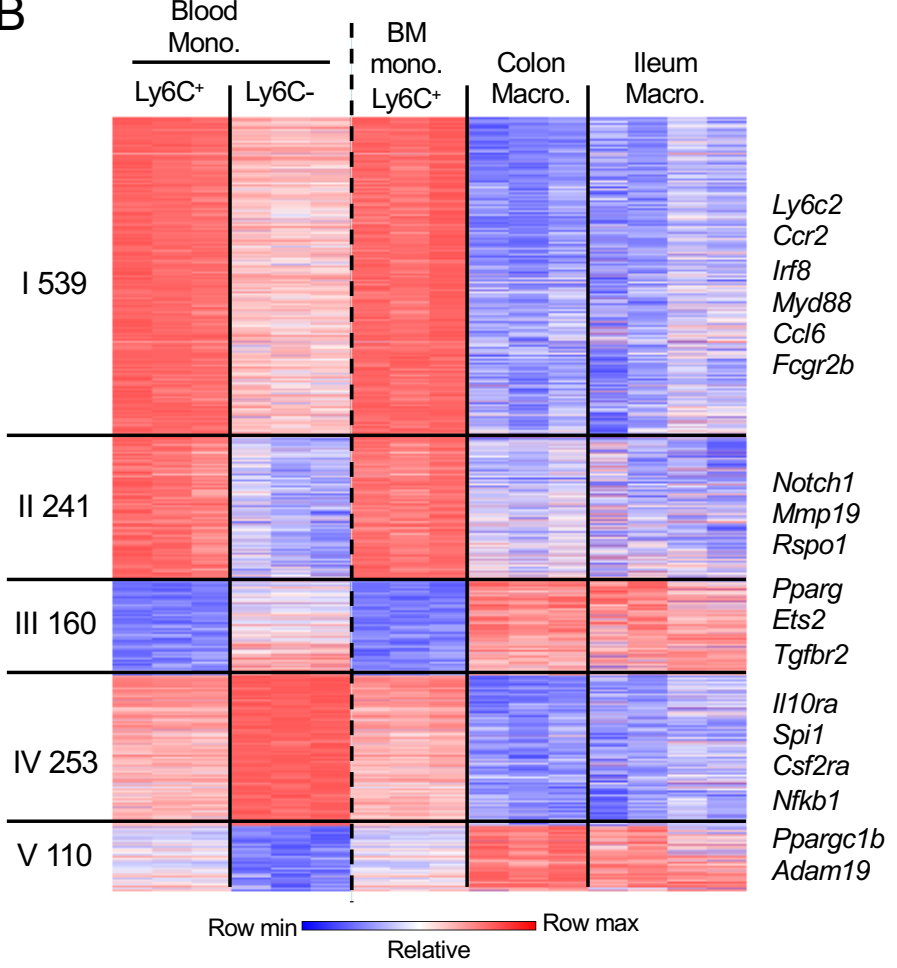

D

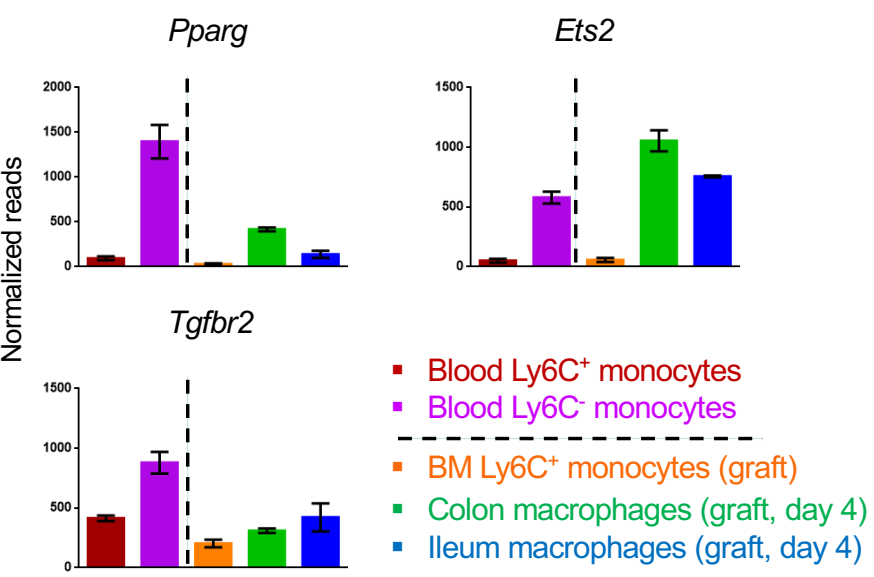

E

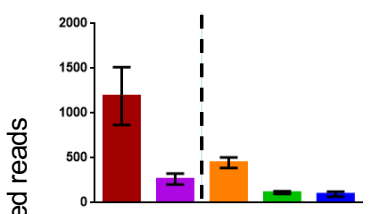

Irf7

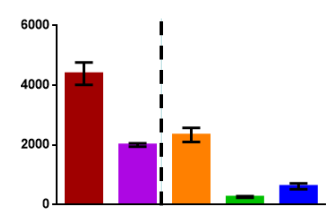

Cebpb

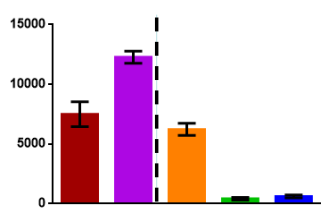

Irf8

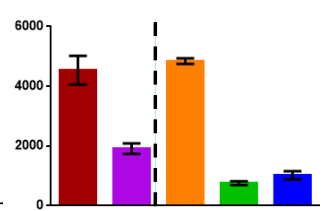

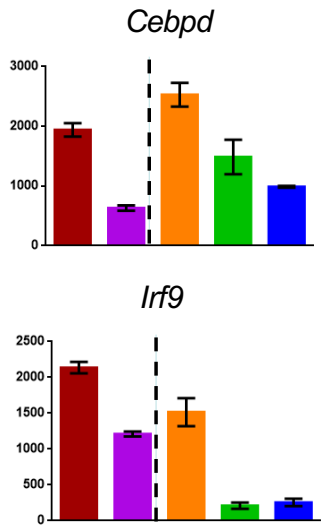


A

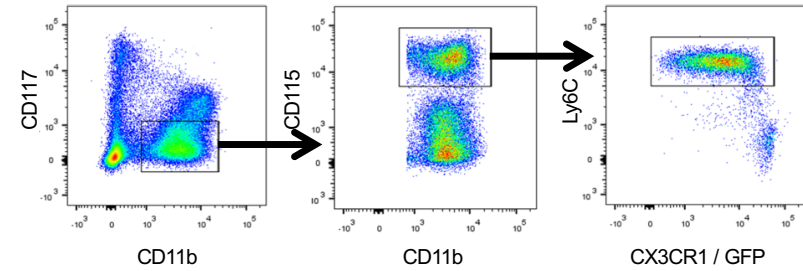

Colon macrophages

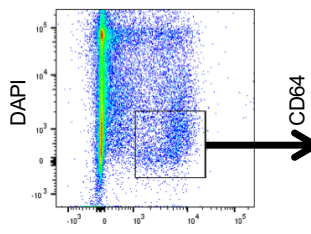

CD45

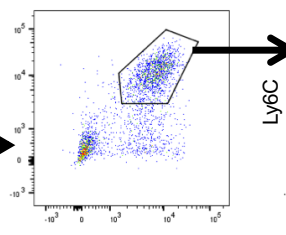

CD11b

B

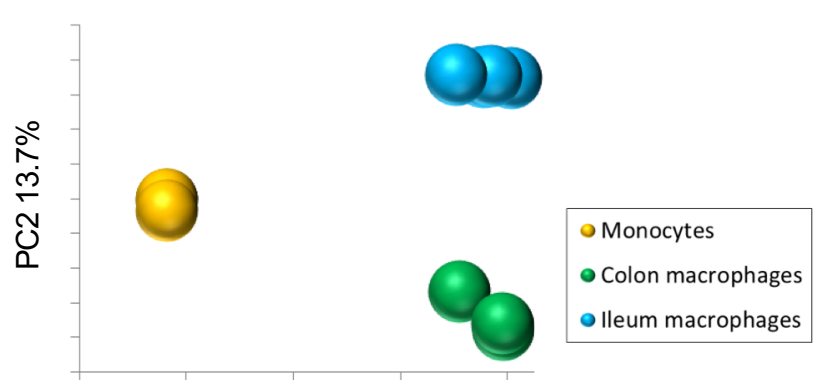

PC1 $70.1 \%$

C

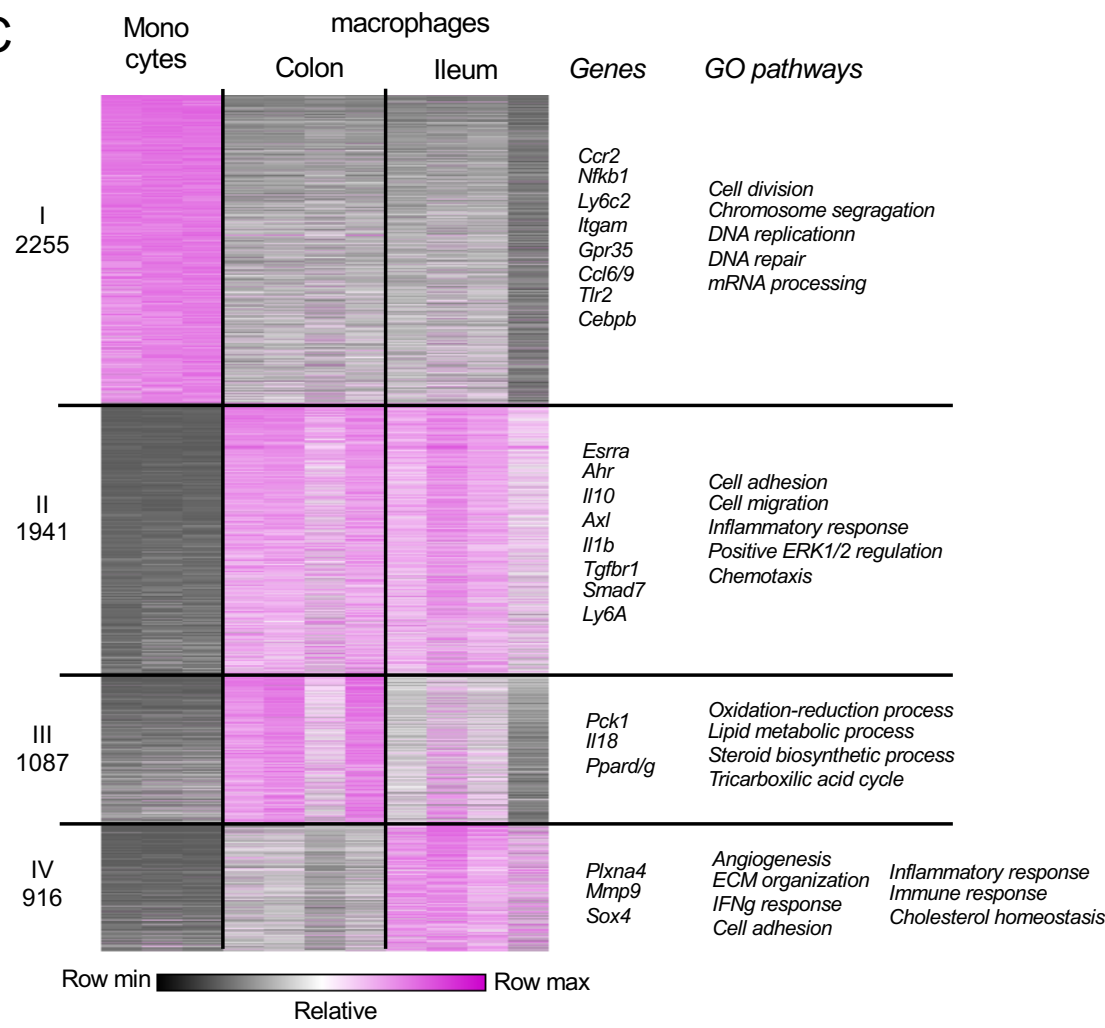

D

\section{Cluster I}

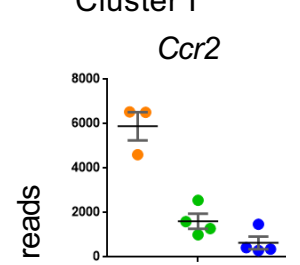

Cluster III

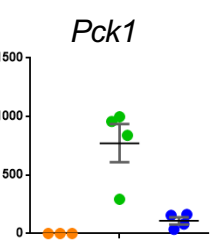

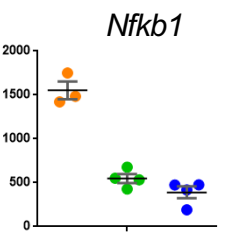

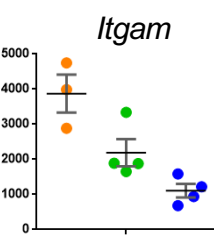

Fcgr1

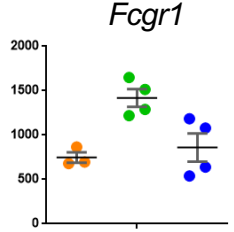

Cluster II

Cluster $\|$
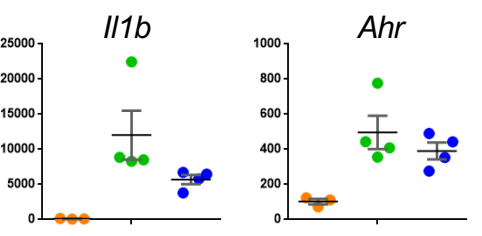

colon macrophages

- ileum macrophages

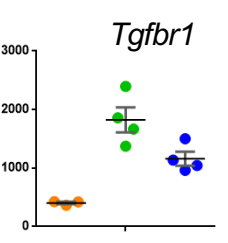

\section{Cluster IV}
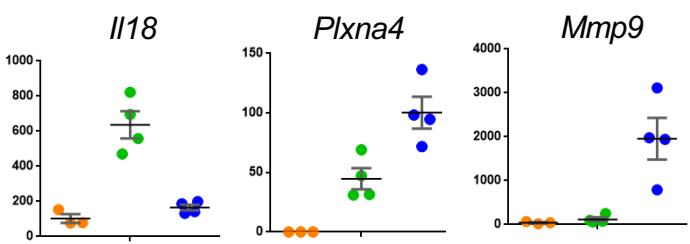
A

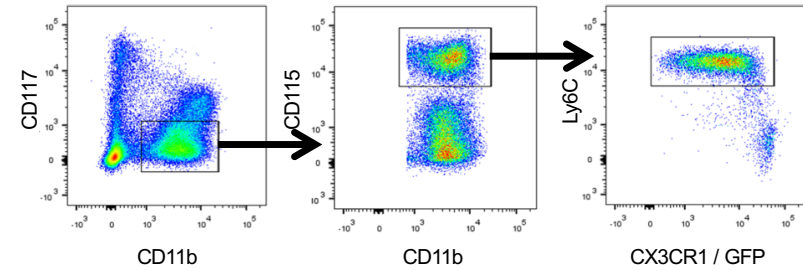

Colon macrophages

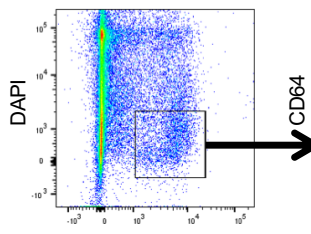

CD45

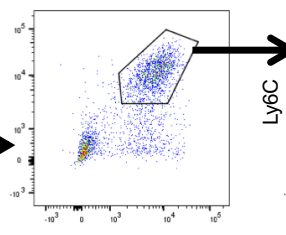

CD11b

B

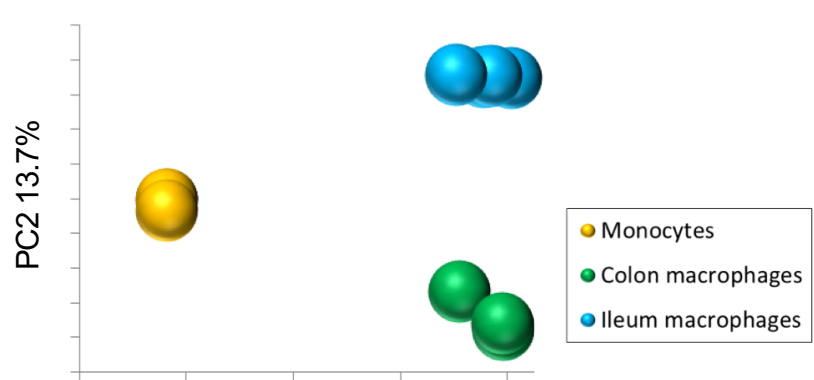

PC1 $70.1 \%$

C

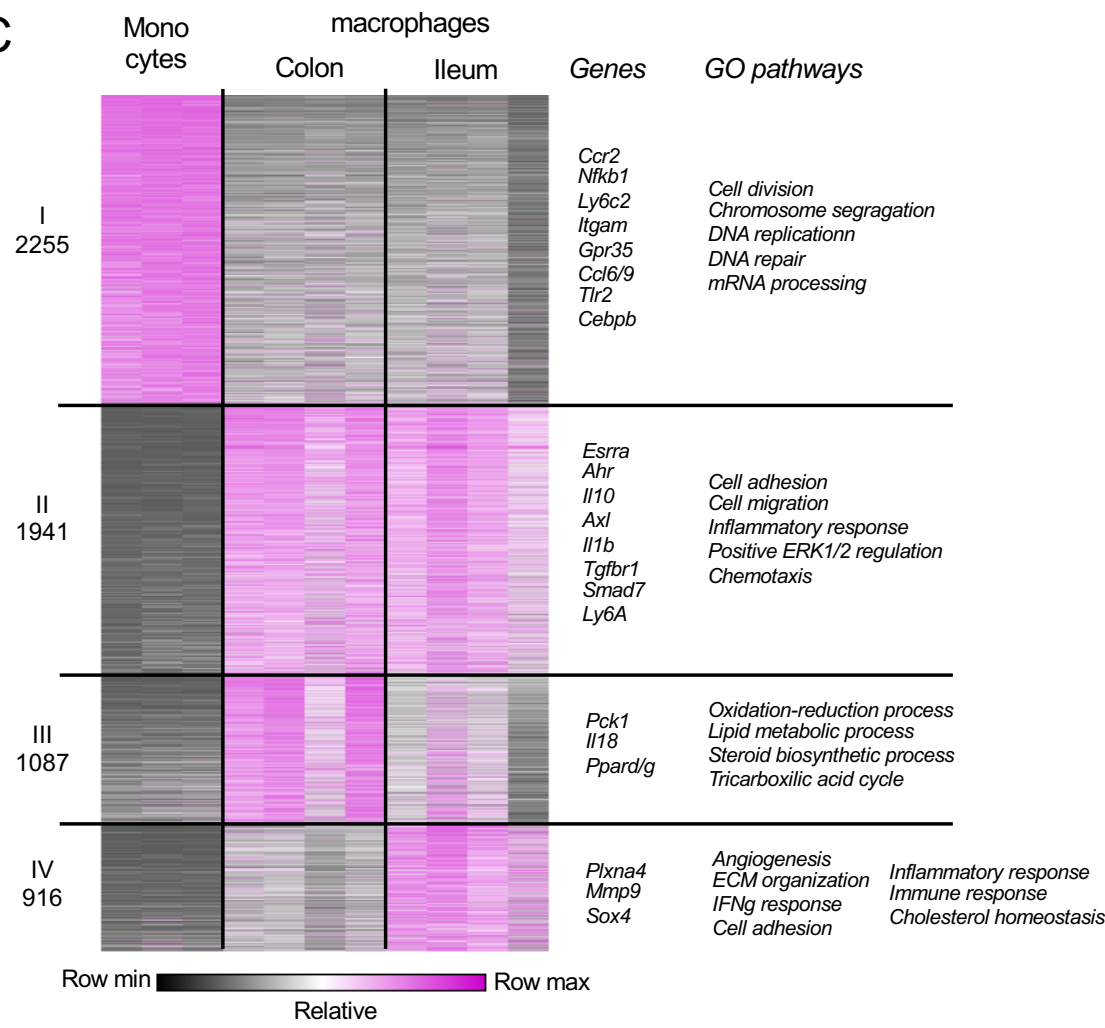

D

\section{Cluster I}

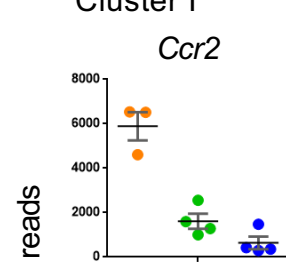

Cluster III

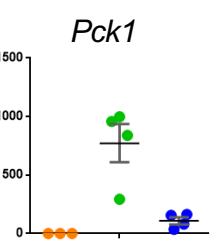

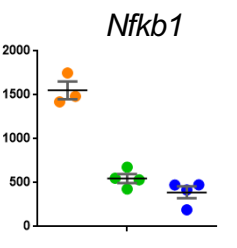

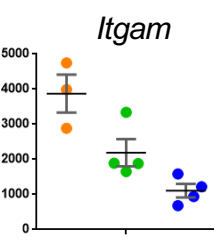

Fcgr1

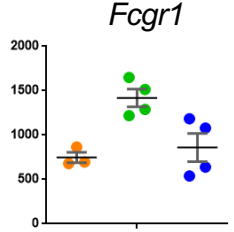

Cluster II

Cluster $\|$
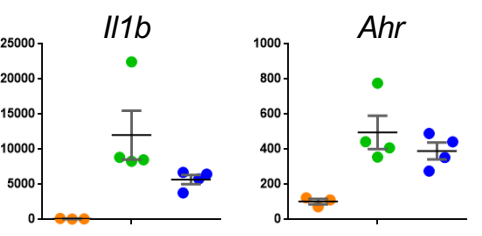

colon macrophages

- ileum macrophages

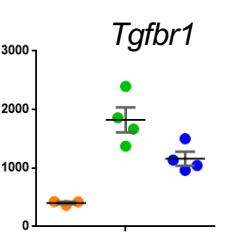

\section{Cluster IV}
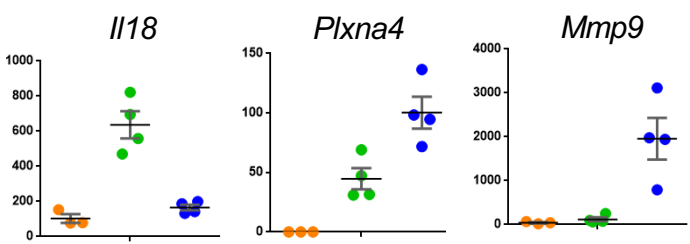
A

$\mathrm{Cx}_{3} \mathrm{CR} 1^{\mathrm{GFP} /+}$ mouse (donor)

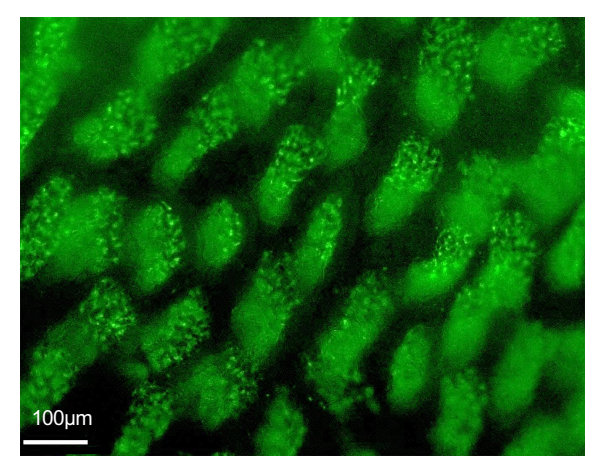

DTx-treated [CD11c-DTR > WT] chimera engrafted with $\mathrm{Cx}_{3} \mathrm{CR} 1^{\mathrm{GFP} /+}$ monocytes

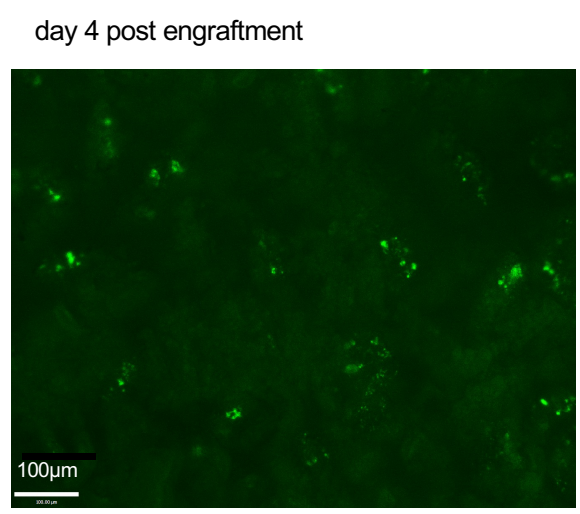

day 8 post engraftment

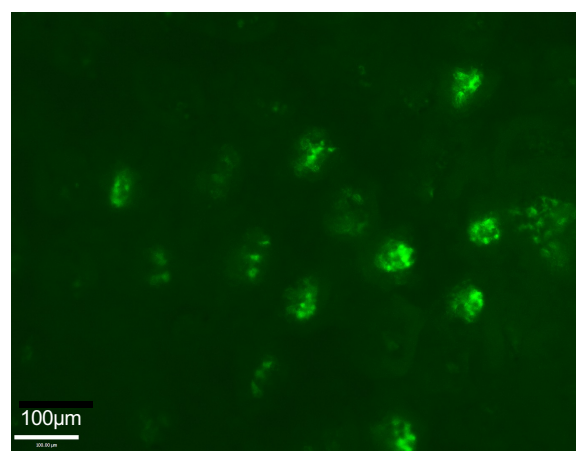

CD $45.2^{+}$host cells

B
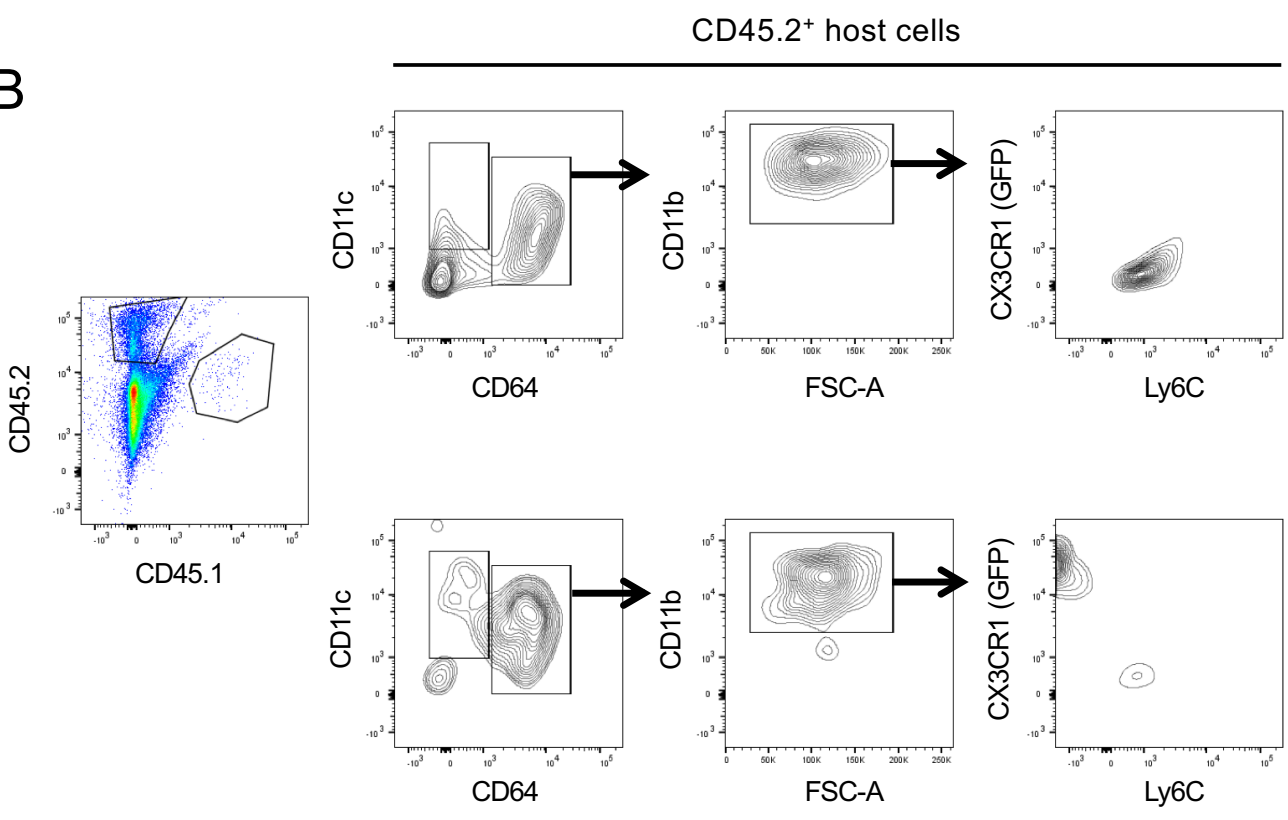

CD45.1+ engrafted cells 
A

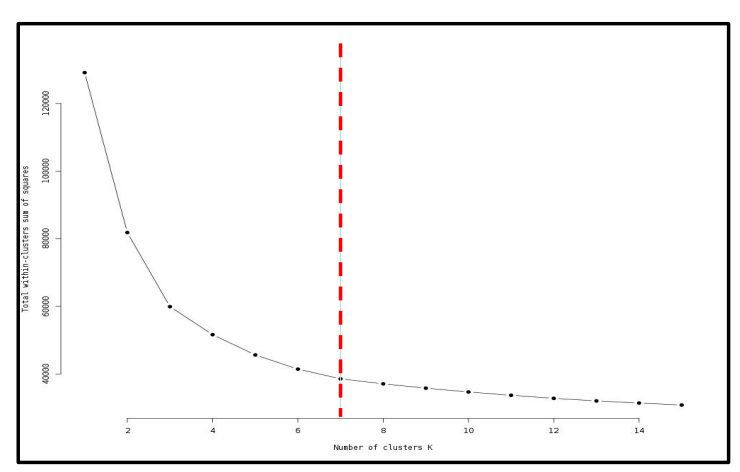

C cluster II
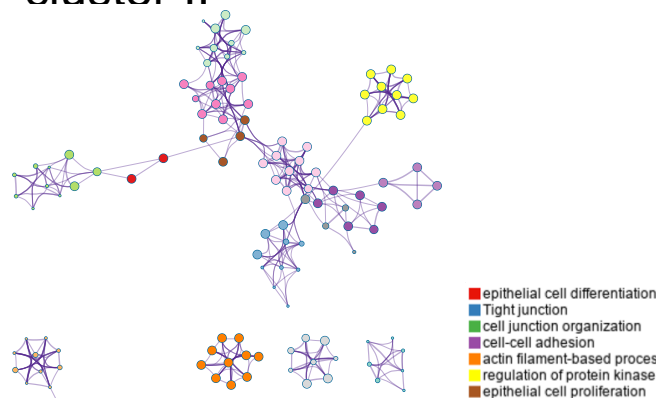

\&

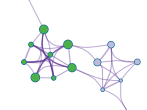

सु०

D cluster III
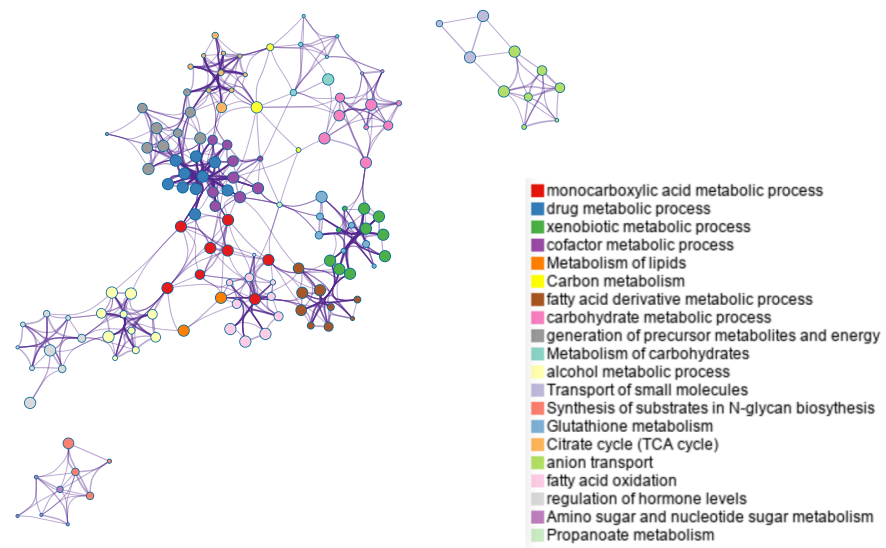

\section{E cluster IV}

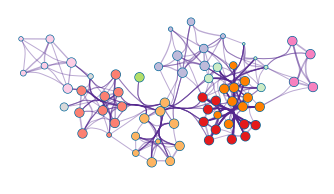

米溇
B
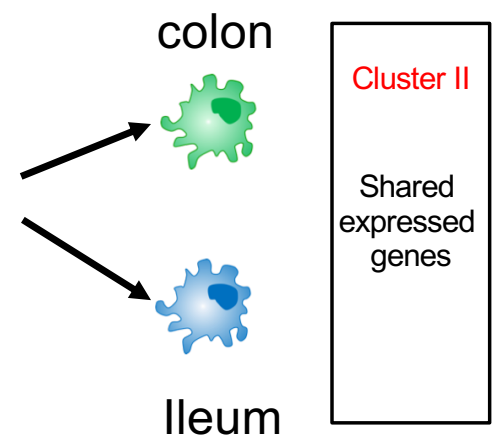

Cluster III preferential

Colon

expression

Cluster IV preferential lleum expression
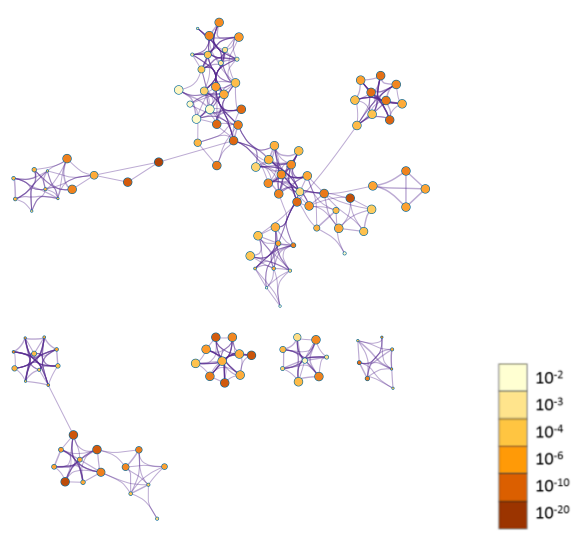

มี :
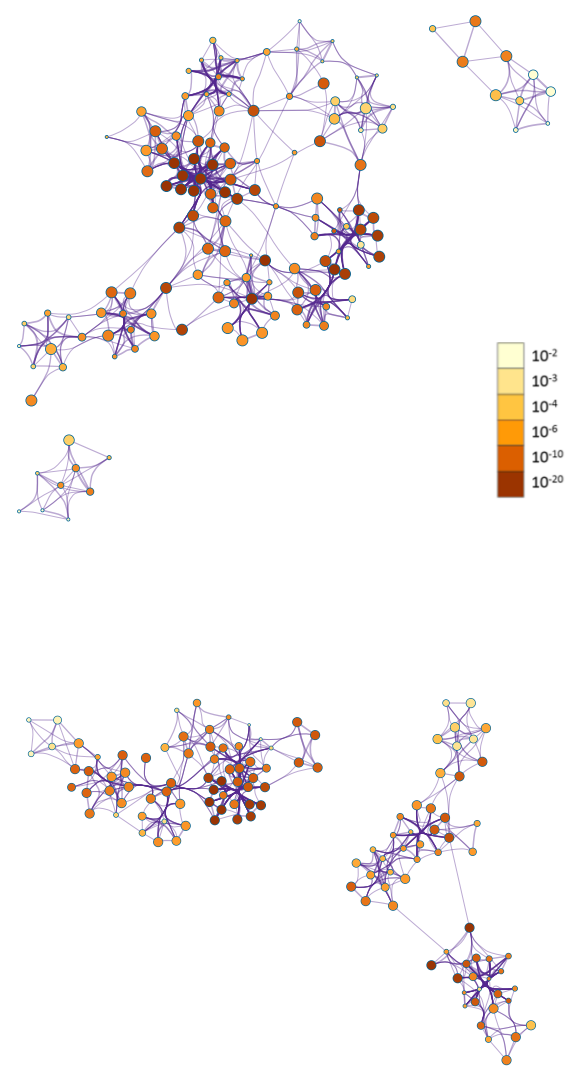

* 粦

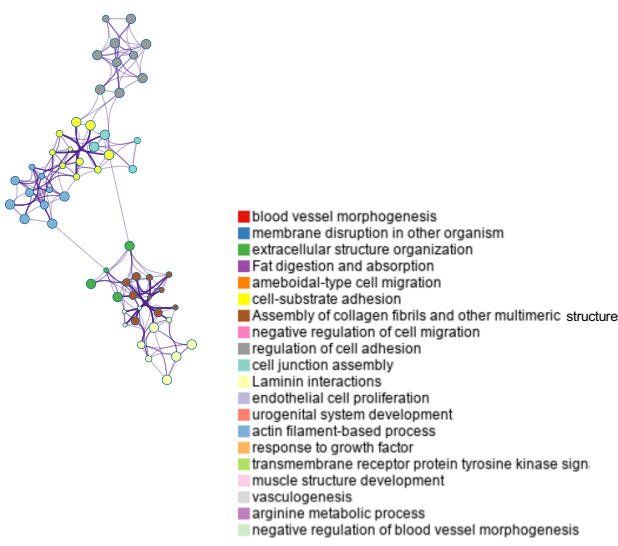



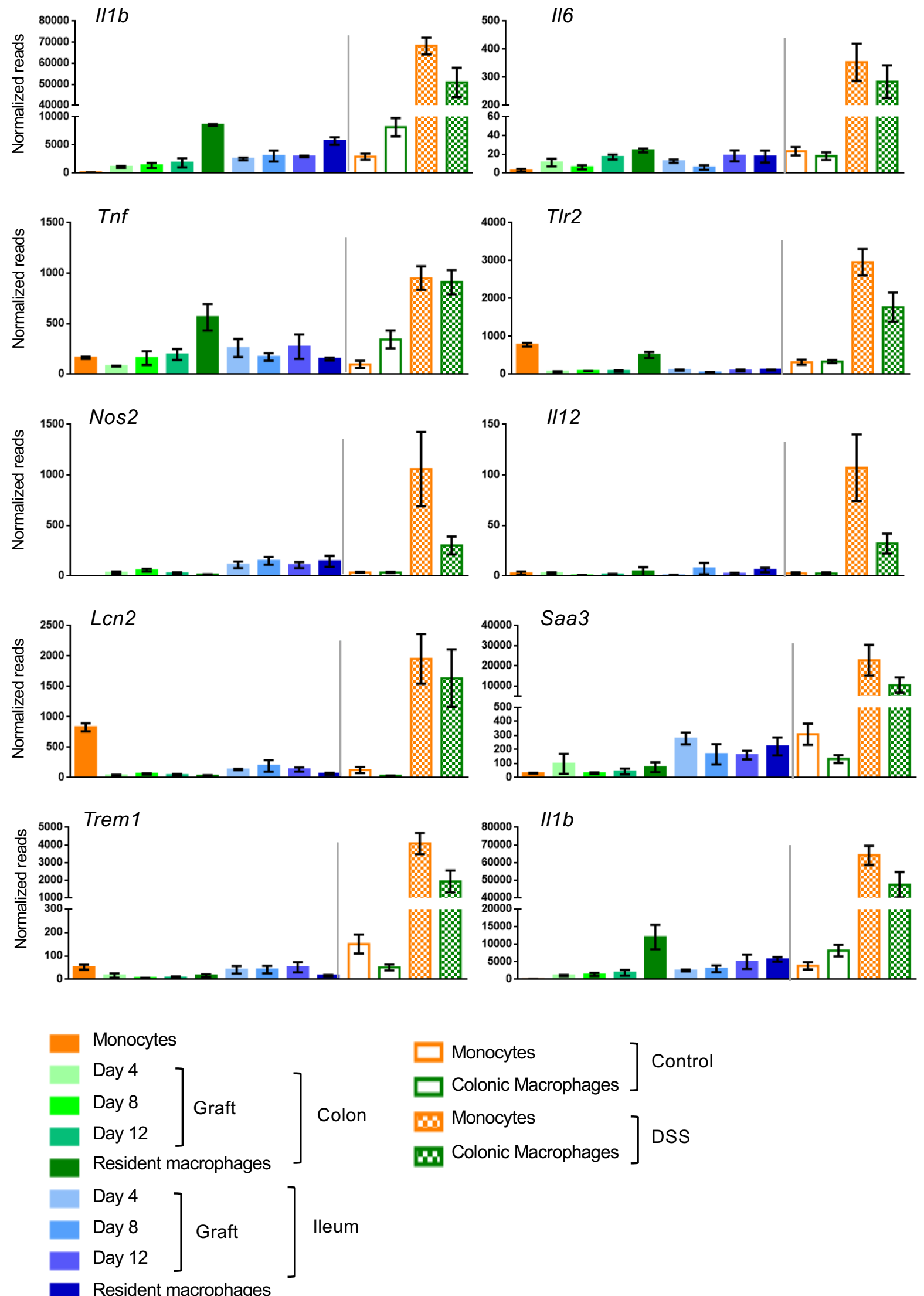

Resident macrophages

Gross-Vered et al. Suppl. Fig. 4 
A
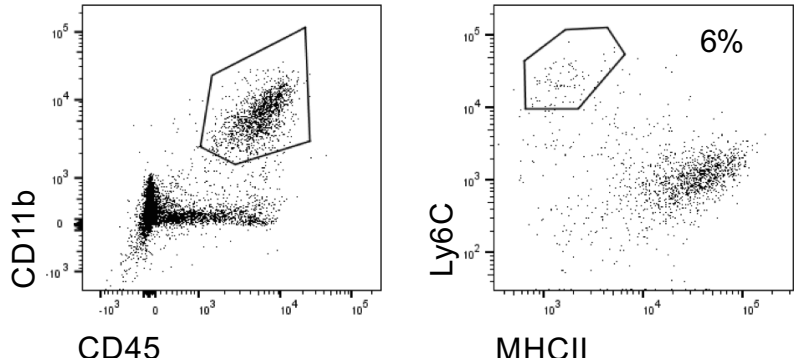

\begin{tabular}{|c|c|c|c|c|c|c|c|c|c|c|c|c|c|c|c|}
\hline \multirow{2}{*}{\multicolumn{2}{|c|}{ B }} & \multicolumn{3}{|c|}{ Ly6C+ BM monocytes } & \multicolumn{3}{|c|}{$\begin{array}{c}\text { Engrafted colon } \\
\text { macrophages day } 4\end{array}$} & \multicolumn{4}{|c|}{ colonic 'monocytes' } & \multicolumn{4}{|c|}{ colonic macrophages } \\
\hline & & \begin{tabular}{|c|} 
Sample \\
1
\end{tabular} & \begin{tabular}{|c|} 
Sample \\
2
\end{tabular} & $\begin{array}{c}\text { Sample } \\
3\end{array}$ & $\begin{array}{c}\text { Sample } \\
1\end{array}$ & \begin{tabular}{|c|} 
Sample \\
2
\end{tabular} & $\begin{array}{c}\text { Sample } \\
3\end{array}$ & \begin{tabular}{|c|} 
Sample \\
1
\end{tabular} & \begin{tabular}{|c|} 
Sample \\
2
\end{tabular} & \begin{tabular}{|c|} 
Sample \\
3
\end{tabular} & $\begin{array}{c}\text { Sample } \\
4\end{array}$ & \begin{tabular}{|c|} 
Sample \\
1
\end{tabular} & \begin{tabular}{|c|} 
Sample \\
2
\end{tabular} & \begin{tabular}{|c|} 
Sample \\
3
\end{tabular} & $\begin{array}{c}\text { Sample } \\
4\end{array}$ \\
\hline \multirow{3}{*}{ Ly6C+ BM monocytes } & Sample 1 & 1.00 & & & & & & & & & & & & & \\
\hline & Sample 2 & 0.98 & 1.00 & & & & & & & & & & & & \\
\hline & Sample 3 & 0.97 & 0.99 & 1.00 & & & & & & & & & & & \\
\hline \multirow{3}{*}{$\begin{array}{l}\text { Engrafted colon } \\
\text { macrophages day } 4\end{array}$} & Sample 1 & 0.48 & 0.45 & 0.43 & 1.00 & & & & & & & & & & \\
\hline & Sample 2 & 0.47 & 0.42 & 0.41 & 0.95 & 1.00 & & & & & & & & & \\
\hline & Sample 3 & 0.47 & 0.41 & 0.41 & 0.90 & 0.97 & 1.00 & & & & & & & & \\
\hline \multirow{4}{*}{ colonic 'monocytes' } & Sample 1 & 0.48 & 0.47 & 0.46 & 0.88 & 0.80 & 0.75 & 1.00 & & & & & & & \\
\hline & Sample 2 & 0.49 & 0.47 & 0.46 & 0.91 & 0.84 & 0.80 & 0.96 & 1.00 & & & & & & \\
\hline & Sample 3 & 0.45 & 0.43 & 0.43 & 0.92 & 0.85 & 0.80 & 0.97 & 0.98 & 1.00 & & & & & \\
\hline & Sample 4 & 0.52 & 0.51 & 0.51 & 0.87 & 0.79 & 0.75 & 0.93 & 0.95 & 0.94 & 1.00 & & & & \\
\hline \multirow{4}{*}{ colonic macrophages } & Sample 1 & 0.44 & 0.43 & 0.44 & 0.68 & 0.64 & 0.60 & 0.79 & 0.77 & 0.75 & 0.78 & 1.00 & & & \\
\hline & \begin{tabular}{|l|} 
Sample 2 \\
\end{tabular} & 0.42 & 0.43 & 0.43 & 0.68 & 0.63 & 0.59 & 0.76 & 0.77 & 0.75 & 0.80 & 0.98 & 1.00 & & \\
\hline & Sample 3 & 0.44 & 0.44 & 0.44 & 0.80 & 0.74 & 0.69 & 0.89 & 0.88 & 0.89 & 0.90 & 0.95 & 0.95 & 1.00 & \\
\hline & Sample 4 & 0.42 & 0.42 & 0.43 & 0.72 & 0.66 & 0.62 & 0.81 & 0.81 & 0.80 & 0.87 & 0.94 & 0.95 & 0.96 & 1.00 \\
\hline
\end{tabular}

C

$N r 3 c 2$

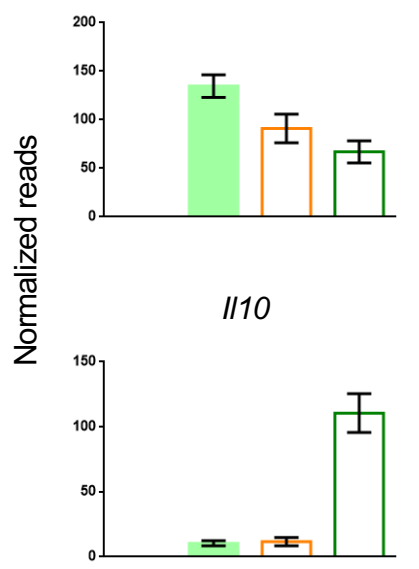

Foxd2

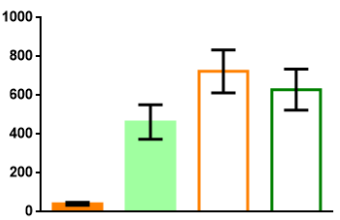

I/10ra

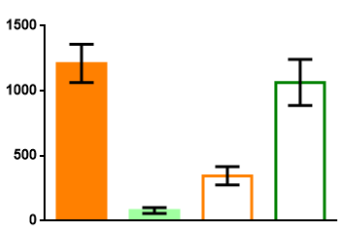

Cd209f

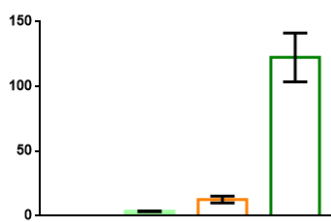

Rp/10

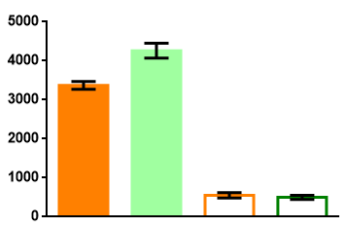

Timd4

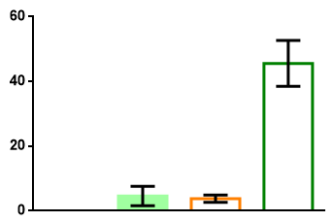

Hmga1

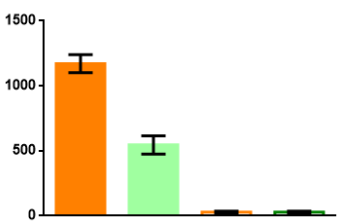

BM Ly6C+ monocytes

engrafted colonic macrophages

colonic 'monocytes'

colonic macrophages

Gross-Vered et al. Suppl. Fig. 5 
A

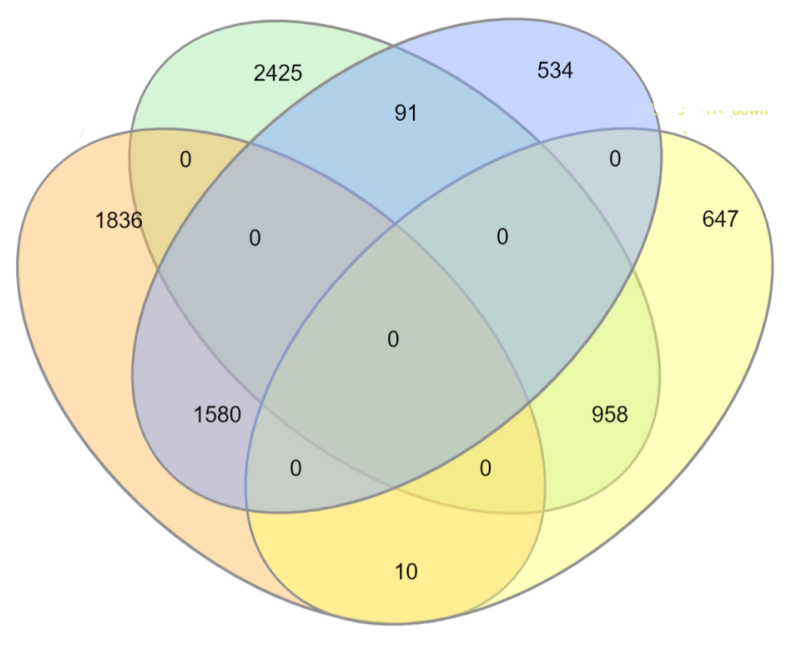

Recipient chimera

[ $x>$ WT]

Cx3Cr1-DTR (up) 3426 genes

Cx3Cr1-DTR (down) 3474 genes

CD11c-DTR (up) 2205 genes

CD11c-DTR (down) 1615 genes

B
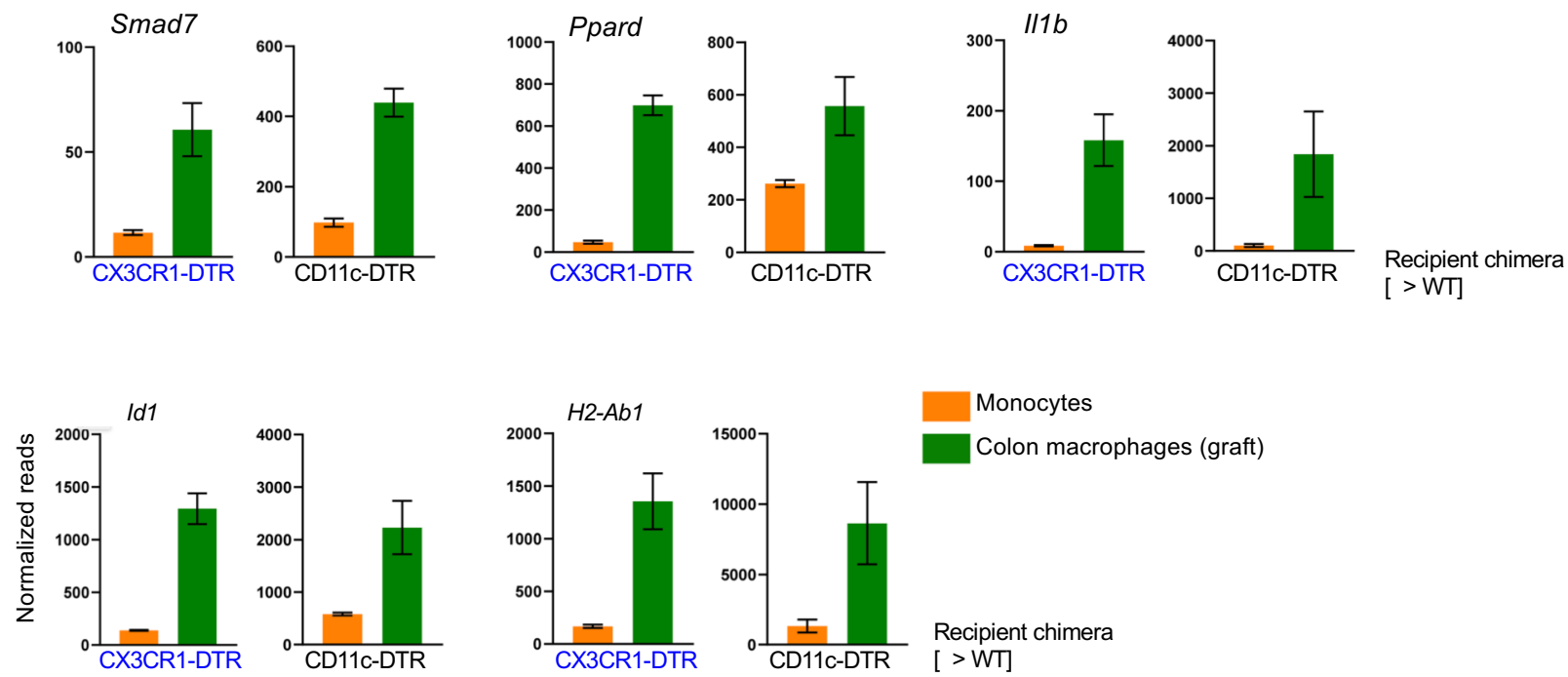

C
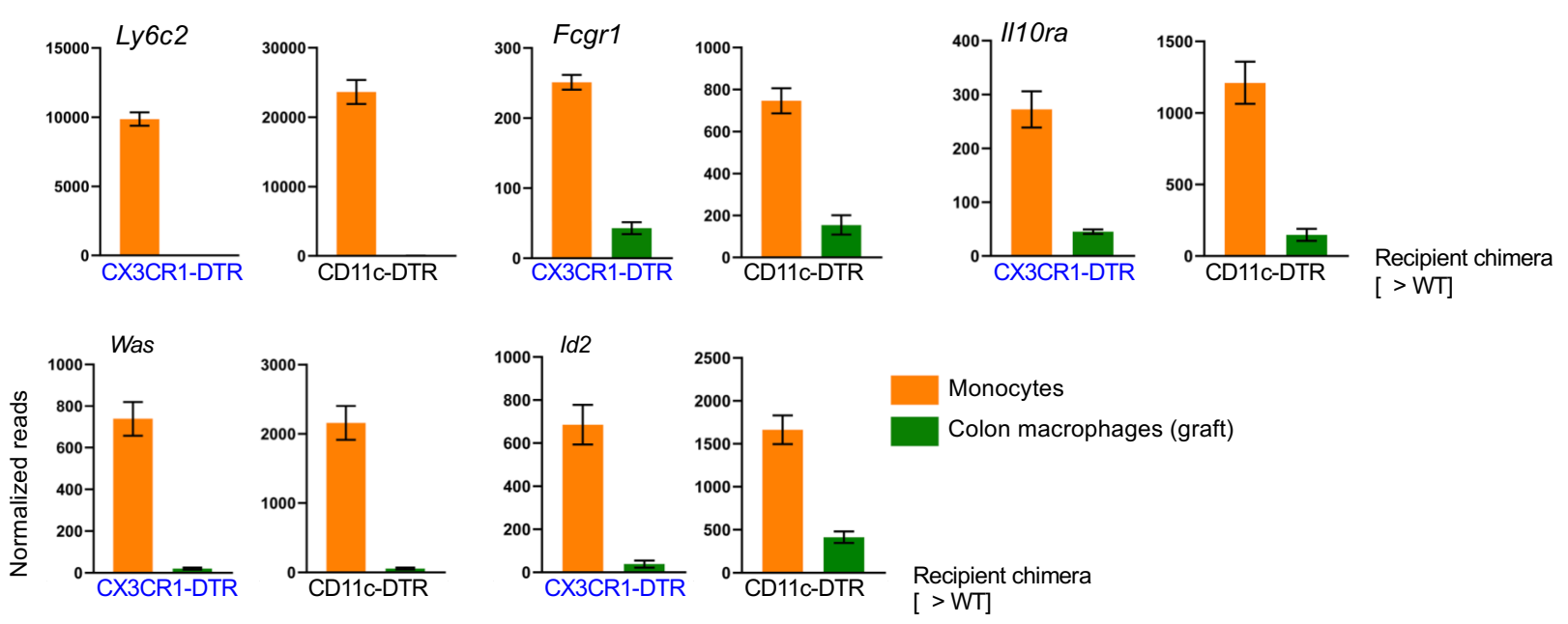


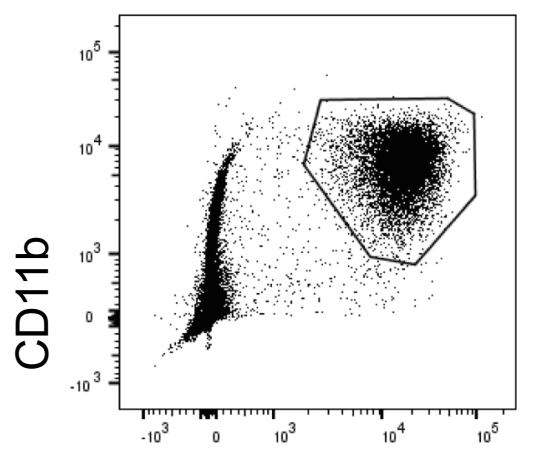

CD115

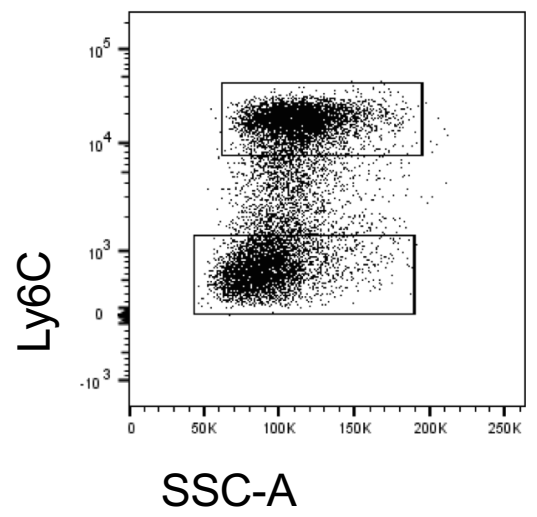

Gross-Vered et al. Suppl. Fig. 7 